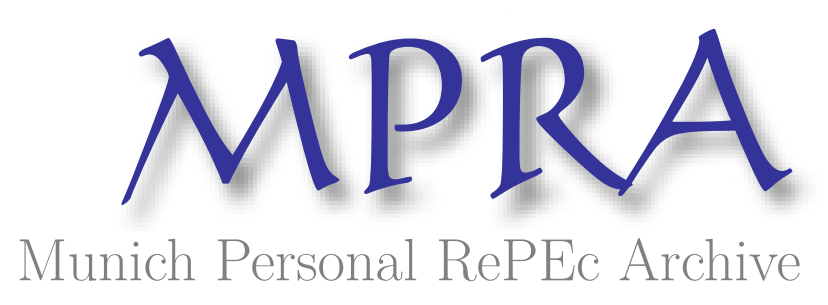

\title{
International Coordination of Debt Rules with Time-inconsistent Voters
}

\author{
Arawatari, Ryo and Ono, Tetsuo \\ Doshisha University, Osaka University
}

20 November 2020

Online at https://mpra.ub.uni-muenchen.de/104274/

MPRA Paper No. 104274, posted 05 Dec 2020 13:13 UTC 


\title{
International Coordination of Debt Rules with Time-inconsistent Voters*
}

\author{
RYO ARAWATARI ${ }^{\dagger}$ \\ Tetsuo OnO \\ Doshisha University \\ Osaka University
}

November 20, 2020

\begin{abstract}
In this study, we investigate the international coordination of debt rules in an economy consisting of a large number of countries with varying degrees of present bias. A case whereby each country sets its own uncoordinated debt rules is compared with a case whereby all countries have common coordinated debt rules. Countries with weak present-biased preferences increase their debt issuance and suffer from welfare losses by participating in coordination. In contrast, countries with strong present-biased preferences reduce their debt issuance and can enjoy welfare improvement by participating in coordination. The contrasting results suggest the possibility that the former have little incentive to follow the coordinated rule.
\end{abstract}

Key words: Debt rules; Debt ceilings; Present bias; International coordination.

JEL Classification: D72, D78, H62, H63

*Financial support from the Japan Society for the Promotion of Science Grants-in-Aid for Scientific Research (C) (No. 17K03620, Ryo Arawatari; No. 18K01650, Tetsuo Ono) is gratefully acknowledged. The authors declare that they have no known competing financial interests or personal relationships that could have appeared to influence the work reported in this paper.

${ }^{\dagger}$ Corresponding author. Faculty of Economics, Doshisha University, Karasuma-higashi-iru, Imadegawa-dori, Kamigyo-ku, Kyoto 602-8580, Japan. E-mail: rarawata@mail.doshisha.ac.jp

${ }^{\ddagger}$ Graduate School of Economics, Osaka University, 1-7, Machikaneyama, Toyonaka, Osaka 560-0043, Japan. Email: tono@econ.osaka-u.ac.jp 


\section{Introduction}

Since the European debt crisis of late 2009, there has been increasing attention to the sustainability of public debt in developed countries (Collignon, 2012; Beqiraj, Fedeli, Forte, 2018). In today's highly integrated world economy, a fiscal crisis in one country could have a ripple effect on other countries. For example, the Greek debt crisis impacted other sovereign bond prices and reduced the financial value of European firms (Bhanot et al., 2014). In the wake of the financial and economic crisis, the EU has placed even greater emphasis on maintaining fiscal discipline in the region and has strictly enforced common fiscal rules to be followed by EU member states (Banerji et al., 2015).

Having a common set of fiscal rules for several countries, such as the EU, is more enforceable in terms of adherence to the rules than if each country has its own fiscal rules. For example, the EU invokes the excessive deficit procedure (EDP) for countries that significantly deviate from the common fiscal rules of the member states, placing the country's finances under EU supervision and in some cases imposing fines (Lledo et al., 2017). The presence of such external sanctions increases the incentive for countries to comply with the rules and makes the fiscal rules more effective.

However, common fiscal rules are not always followed. The financial crisis of early 2010, for example, was caused by the incoming Greek government's revelation that the budget deficit reported in 2009 was underestimated (Higgins and Klitgaard, 2011). This suggests that Greece may not have followed the EU's rule of keeping the budget deficit within 3 percent of GDP. Italy was also pointed out by the EU in 2019 for its failure to comply with the rule mentioned above (European Commission, 2019). ${ }^{1}$ Although the EDP was not triggered in either case, these cases illustrate the difficulty in getting all the member countries to comply with the common rules.

In this background, this study addresses three fundamental questions: 1. Which common fiscal rule is optimal under the possibility of violation of the rule? 2. Which countries would benefit (or lose) from the introduction of a common fiscal rule? 3. Would a common fiscal rule improve fiscal discipline in all participating countries? To answer these questions, this study provides a theoretical analysis using a political economy model. For the common fiscal rule, this study focuses on the ceiling on government debt.

\footnotetext{
${ }^{1}$ According to EEAG (2011), such rule violations are not uncommon and occur frequently in EU member countries.
} 
In considering the role of debt rules, this study focuses on time-inconsistent decision-making arising from households' present-biased preferences. If households' preferences have a present bias, they have an incentive to consume more in the present compared to their previous consumption plans, which they previously considered optimal (Strotz, 1956; Phelps and Pollak, 1968; Laibson, 1997). In addition, present-biased preferences also affect the formation of fiscal policies. Households can increase current consumption and decrease future consumption by voting to support the issuance of public bonds and tax cuts. Such voting behavior suggests the need for a rule imposing a cap on public bond issuance.

Bisin, Lizzeri, and Yariv (2015) focus on the impact of households' presentbiased preferences on fiscal policy formation. In their three-period model, households gain consumption in the second and third periods by using the resources they hold in the first period to purchase liquid and illiquid assets. Households in period 2 (hereafter, period-2 selves) with present-biased preferences will have incentives to consume more in the second period and less in the third period. Such a change in plans is undesirable for period-1 households (hereafter, period-1 selves). Therefore, period-1 selves attempt to prevent overconsumption by period- 2 selves through the purchase of illiquid assets. However, period-2 selves can increase their consumption in the second period by cutting taxes and issuing bonds through voting fiscal policy choices. If period-1 selves do not have sufficient control over period-2 selves' government bond issuance through asset selection, then the ceiling on government bond issuance becomes effective.

Arawatari and Ono (2020a) extend the model of Bisin, Lizzeri, and Yariv (2015) by assuming that households can determine the government debt ceiling in the first period through a vote and that they can choose debt issuance above the ceiling by paying additional costs. These settings allow us to investigate why fiscal rule violations, reported in Wyplosz (2013), occur frequently. We extend the model further to an economy consisting of a large number of countries with varying degrees of present bias. This extension allows us to consider the formation of a common debt rule among countries (henceforth, a coordinated debt rule), which aims to maximize the weighted sum of utility functions of all countries. In particular, this study focuses on the mandatory case in which all countries are forced to form a coalition, regardless of whether they benefit or lose from the coordinated debt rule. The extension also enables us to compare the coordinate rule to an uncoordinated debt rule that aims to maximize each country's welfare. The goal of our analysis is to measure the changes in government bond issuance and welfare of each country when moving from an uncoordinated rule case to a 
coordinated rule case.

The results of this study are threefold. First, we compare the government debt ceiling set by period- 1 selves in the case of the uncoordinated rule with that of the coordinated rule. Whether a case achieves a stricter ceiling on government bond issuance depends on the degree of present bias in each country (Propositions 2 and 3). In the uncoordinated rule case, each country sets an optimal debt ceiling according to the level of its present bias. If the ceiling on government bond issuance is set at a low level, period-2 selves will have an incentive to vote for government bond issuance over the ceiling. To avoid such rule-breaking, period-1 selves set the ceiling high enough to ensure that period-2 selves do not break the rule. Meanwhile, under the coordinated rule, all countries are subject to the same ceiling on government bond issuance. Thus, the ceiling in the coordination rule case is tighter for countries with a strong present bias and, conversely, looser for countries with a weak present bias.

Second, we compare the volume of government bond issuance under the uncoordinated rule with that under the coordinated rule. Whether a case achieves a higher bond issuance depends on the degree of present bias (Proposition 4). As noted above, for countries with strong present-biased preferences, the debt ceiling is tighter under the coordinated rule than under the uncoordinated rule. This means that countries with strong present-biased preferences will reduce their bond issuance by participating in the coordinated rule. However, for countries with weak present-biased preferences, the ceiling on government bond issuance is looser under the coordinated rule than under the coordinated rule. Thus, countries with weak present-biased preferences will increase their bond issuance by participating in the coordinated rule.

Finally, whether countries achieve improvements in social welfare by participating in the coordinated rule depends on the following three effects: 1 . The unifying effect, which requires all countries to have the same debt ceiling. This causes welfare losses as it leads to deviations from the optimal debt ceiling for each country. 2. The penalty effect of deviating from the coordinated rule. This penalty weakens the incentive for each country to deviate from the coordinated rule, reduces the volume of government bond issuance, and improves welfare. 3 . The distortionary effect, where countries with a high degree of present bias, even with the penalties described above, choose to deviate from the coordinated rule and issue bonds over the ceiling. The costs associated with this will result in welfare losses. The functioning of any of these three effects depends on the degree of present bias. In countries with weak present-biased preferences, participation in 
the coordinated rule will lead to a deterioration in social welfare as a result of the unifying effect. By contrast, in countries with strong present-biased preferences, the net effect of participating in the coordination on social welfare depends on the degree of present bias as well as other structural parameters, as the remaining two effects, in addition to the unifying effect, also operate (Proposition 5).

The first and second results lead to the consequence that the introduction of the coordinated rule works to reduce the volume of bond issuance in countries with strongly present-biased preferences and excessive debt. In other words, the coordinated rule is more effective than the uncoordinated rule in achieving the objective of curbing the excessive issuance of public debt and restoring fiscal health. However, if participation in the coordinated debt rule is optional rather than mandatory for each country, then, as the third result suggests, whether countries are willing to adopt the coordinated rule depends on the relative size of the three effects mentioned above. In particular, when the unifying and distortionary effects dominate the penalty effect, countries with strongly present-biased preferences and excessive debt will refuse to adopt the coordinated rule. This implies the difficulty of introducing and maintaining the coordinated rule that contributes to the fiscal health of regional economies such as the EU . Therefore, to get countries to commit to the coordinated rule, it will be necessary to implement measures such as the acceptance of standard violations in times of economic downturn or broader cooperation among countries on fiscal issues.

This study is related to the literature on the political economy of fiscal rules (Arawatari and Ono, 2020a; Coate and Milton, 2019; Dovis and Kirpalani, 2020; Halac and Yared, 2018). All of which (except Halac and Yared, 2018) analyze the formation of fiscal policy in the case of possible violations of fiscal rules. As their analyses focus on a single domestic fiscal rule, they do not address the international coordination of fiscal rules. In contrast, Halac and Yared (2018) present an open economy model in which they compare two sets of rules: coordinated rules chosen jointly by a group of countries and uncoordinated rules chosen independently by each country. However, they do not provide for potential violations of fiscal rules. They show that the coordinated rules are slacker when the present bias is large because of a disciplining effect through the interest rate. This study abstracts away such an effect through the interest rate but unlike Halac and Yared 2018, provides for the possibility of rule violation in each country, as in Coate and Milton (2019) and Dovis and Kirpalani (2020). We show that this possibility shapes rule formation critically, and the resulting fiscal discipline is tighter in the coordinated case than in the uncoordinated case. Our results are in contrast to those shown 
in Halac and Yared (2018).

This study is also related to the literature on the international political economy of public debt. This literature can be divided into two categories: the study of small open economies (e.g., Persson and Svensson, 1989; Song, Storesletten, and Zilibotti, 2012; Arawatari and Ono, 2017, 2020b) and the study of large open economies (e.g., Chang, 1990; Arcalean, 2017, 2018; Janeba and Todtenhauput, 2018). This study is in line with the first category in that the policy of each country is assumed to have no direct impact on the world interest rate. This study is also in line with the second category in that we compare debt and welfare associated with uncoordinated and coordinated fiscal policies and examine under which conditions welfare is improved by international policy coordination. None of the above-mentioned studies address the international coordination of fiscal rules, whereas this study focuses on its role.

The remainder of this paper is organized as follows: Section 2 presents the model. Section 3 characterizes an equilibrium allocation for a given debt rule. Section 4 considers the endogenous determination of debt rules via voting. In particular, we examine two types of rules, the uncoordinated rule and the coordinated rule, and compare them in terms of debt levels and welfare. Section 5 provides concluding remarks. All proofs are presented in the Appendix.

\section{The Model}

The model is based on the one developed by Bisin, Lizzeri, and Yariv (2015). It measures identical agents who live for three periods, 1, 2, and 3. They are endowed with $k$ units of goods in period 1 and nothing in periods 2 and 3 . In period 1, agents only make savings and portfolio decisions; they receive utility from consumption in periods 2 and 3 .

Agents (hereafter interchangeably called individuals, selves, and voters) have time-inconsistent, present biased preferences (Stortz, 1965; Phelps and Pollak, 1968; Laibson, 1997). In particular, agents' preferences over consumption in period 2 and $3, c_{2}$ and $c_{3}$, are given by the following utility functions:

$$
\begin{aligned}
U_{1}\left(c_{2}, c_{3}\right) & =\beta\left[u\left(c_{2}\right)+u\left(c_{3}\right)\right], \\
U_{2}\left(c_{2}, c_{3}\right) & =u\left(c_{2}\right)+\beta u\left(c_{3}\right),
\end{aligned}
$$

where $U_{t}(t=1,2)$ is the assessed utility at time $t, u$ is a continuous and strictly concave utility function, and $\beta \in(0,1)$ is a parameter representing the degree of 
present bias; a lower $\beta$ implies that period-2 selves are biased toward more period2 consumption. Agents are assumed to be sophisticated; they are fully aware of their self-control problems.

Agents choose to invest their wealth, $k$, in liquid or illiquid assets in period 1. It is assumed that all liquid and illiquid assets have the same exogenous interest rate of zero. Liquid assets are one-period securities that are sold in period $t$ $(t=1,2)$ and redeemed in period $t+1$. Illiquid assets are two-period securities that are sold in period 1 and redeemed in period 3; they cannot be sold in period 2. Savings in one- and two-period securities in period 1 are denoted by $s_{12}$ and $s_{13}$, respectively; the subscript $i j$ is the time of saving, $i$, and redemption, $j$. In period 2, agents can save the return from $s_{12}$ in one-period securities; this saving is denoted by $s_{23}$.

Agents displaying present-biased preferences suffer from self-control problems. In particular, period-2 selves are tempted to increase consumption in period 2 at the cost of reduced consumption in period 3. Period-1 selves use illiquid assets to constrain the consumption plans of their future selves. However, the government, representing period-2 selves, is induced to issue public debt in the international market to respond to period-2 selves' desire to undo the commitment made in period 1. This gives sophisticated agents an incentive to rebalance their portfolios in period 1 to reestablish their consumption sequence commitment. This, in turn, creates a demand for further debt accumulation. The debt issue, denoted by $d$, is assumed to be costly and constrained by the constitutionally imposed debt ceiling, denoted by $\bar{d}$, but debt issues beyond the ceiling are available by incurring some additional costs, as specified below:

The budget constraints in periods 1,2 , and 3 are given by

$$
\begin{aligned}
& \text { period 1: } s_{12}+s_{13} \leq k, \\
& \text { period 2: } c_{2} \leq s_{12}+d-s_{23}, \\
& \text { period 3: } c_{3} \leq s_{13}+s_{23}-G(d),
\end{aligned}
$$

where $G(d)$ represents the costs of debt repayment, specified as follows:

$$
G(d)= \begin{cases}(1+\eta) d & \text { when } d \leq \bar{d} \\ (1+\eta) d+(\gamma+\omega)(d-\bar{d}) & \text { when } d>\bar{d}\end{cases}
$$

where $\eta>0, \gamma>0$, and $\omega \geq 0$.

Debt is financed by foreign lenders at an interest rate of zero but can be directly 
distortionary. The term $\eta$ represents the marginal cost of debt issuance, such as labor supply distortions induced by increased tax burdens for debt repayments (Bisin, Lizzeri, and Yariv, 2015). The term $\gamma$, introduced in this study, represents the marginal costs of issuing public debt, conditional on the level of debt being above $\bar{d}$. Such costs could be viewed as reputational losses for rule-breaking counties (Eyraud et al., 2018). The term $\omega$ presents the penalties for rule violation when a country violates an internationally accepted debt ceiling (Yared, 2019). For example, in the EU, an excessive deficit procedure applies whenever a fiscal limit is breached. The procedure is costly and imposes potential sanctions (Lledo et al., 2017). Thus, the marginal cost of debt issuance beyond the ceiling, $\bar{d}$, is $\gamma$ when each country sets the ceiling independently, and it is $\gamma+\omega$ when countries agree on and share a common ceiling. We assume that the costs of debt issuance and rule-breaking, expressed in $\eta$ and $\gamma$, respectively, are bounded, and we also assume that the penalties, expressed in $\omega$, are capped as in Dovis and Kirpalani (2020) and Halac and Yared (2020). Otherwise, regardless of the choice of the debt ceiling, an efficient allocation, defined below, will always be achieved.

The timing of events and the optimization problem at each stage are as follows: In period 1 , an agent who predicts an equilibrium per capita public debt level of $d$, chooses period-1 savings intended for period $2, s_{12}$, and for period $3, s_{13}$, to maximize the assessed utility in period $1, U_{1}$. As the debt level is determined by the government representing period-2 selves, each agent takes it as given when making his/her saving decision. The problem of period-1 selves is

$$
\begin{array}{ll}
\max _{s_{12}, s_{13}} & \beta\left[u\left(s_{12}+d^{e}-s_{23}\left(s_{12}\right)\right)+u\left(s_{13}+s_{23}\left(s_{12}\right)-G\left(d^{e}\right)\right)\right] \\
\text { s.t. } & s_{12}+s_{13} \leq k, \\
& s_{12} \geq 0, s_{13} \geq 0, \\
& \text { given } d^{e},
\end{array}
$$

where $d^{e}$ denotes the expected level of debt issue in period 2, which is taken as given. The term $s_{23}\left(s_{12}\right)$ implies that agents know that their choice of $s_{12}$ (and thus $s_{13}$ ) will have an effect on period-2 saving choice, $s_{23}$. Private borrowing is not allowed, following Bisin, Lizzeri, and Yariv (2015).

In period 2 , an agent chooses the savings intended for period $3, s_{23}$, taking $d^{e}$ as given, to maximize the assessed utility in period 2. The problem of period-2 
selves is

$$
\begin{array}{ll}
\max _{s_{23}} & u\left(s_{12}+d^{e}-s_{23}\right)+\beta u\left(s_{13}+s_{23}-G\left(d^{e}\right)\right) \\
\text { s.t. } & s_{23} \geq 0, \\
& \text { given } s_{12}, s_{13}, \text { and } d^{e} .
\end{array}
$$

The government, representing period-2 selves, chooses public debt issue, $d$, to maximize the utility of period2 selves, subject to a non-negativitiy constraint, $d \geq 0$, and a constitutionally imposed debt ceiling, $\bar{d}$, given $s_{12}, s_{13}$, and $s_{23}{ }^{2}$

$$
\begin{array}{ll}
\max _{d} & u\left(s_{12}+d-s_{23}\right)+\beta u\left(s_{13}+s_{23}-G(d)\right) \\
\text { s.t. } & d \geq 0, \\
& \text { given } s_{12}, s_{13}, \text { and } s_{23} .
\end{array}
$$

Figure 1 illustrates the timing of events.

[Figure 1 is here.]

For our analysis, we make the following assumptions. First, the utility function is specified as

$$
u(c)=\ln c .
$$

This assumption enables us to solve the model analytically. Second, the borrowing must be below the natural debt limit, $k / \eta$, to prevent the government from defaulting. Third, to define the debt ceiling, it is assumed that the ceiling is below the natural debt limit, as in the following assumption. ${ }^{3}$

Assumption 1. $\bar{d}<k / \eta$.

We characterize the first-best allocation as a benchmark. The first-best allocation, denoted by $\left(c_{2}^{f}, c_{3}^{f}\right)$ is the optimal consumption sequence with commitment in period 1. Namely, $\left(c_{2}^{f}, c_{3}^{f}\right)$ maximizes $\ln c_{2}+\ln c_{3}$ subject to the resource constraint, $c_{2}+c_{3} \leq k$. Thus, it is optimal for the period-1 selves to allocate resources evenly between the two periods, $\left(c_{2}^{f}, c_{3}^{f}\right)=(k / 2, k / 2)$. We note that the debt issue, $d>0$, leads to an inefficient allocation of resources because it reduces the available resource for consumption. In other words, $d=0$ is necessary for an allocation to be efficient.

\footnotetext{
${ }^{2}$ Lending in the international market, $d<0$, is abstracted away from the analysis because our focus is on borrowing, $d>0$. Allowing for $d<0$ does not qualitatively alter the following result.

${ }^{3}$ This assumption works when we solve the period-1 agents' optimization problem.
} 


\section{$3 \quad$ Fiscal Policy Making}

As mentioned above, agents are assumed to be sophisticated. Thus, we solve the model through backward induction; that is, we first solve the government's problem in period 2, the agents' problem in period 2, and finally, the agents' problem in period 1. Our results would not change if the timing within period 2 is reversed because the period- 2 selves and the government share a common objective.

\subsection{Government's Decision in Period 2}

The objective of the government, representing the period- 2 selves, is

$$
V_{g}\left(s_{12}, s_{23}, d\right) \equiv \ln \left(s_{12}+d-s_{23}\right)+\beta \ln \left[k-s_{12}+s_{23}-G(d)\right] .
$$

Assuming interior solutions, we can write down the first-order conditions with respect to $d$ when $d \leq \bar{d}$ and $d>\bar{d}$, as follows:

$$
\begin{aligned}
\left.\frac{\partial V_{g}\left(s_{12}, s_{23}, d\right)}{\partial d}\right|_{d \leq \bar{d}}= & \left(s_{12}+d-s_{23}\right)^{-1}-\beta(1+\eta) \cdot\left[k-s_{12}+s_{23}-(1+\eta) d\right]^{-1}, \\
\left.\frac{\partial V_{g}\left(s_{12}, s_{23}, d\right)}{\partial d}\right|_{d>\bar{d}}= & \left(s_{12}+d-s_{23}\right)^{-1} \\
& -\beta(1+\eta+\gamma+\omega) \cdot\left[k-s_{12}+s_{23}-(1+\eta+\gamma+\omega) d+(\gamma+\omega) \bar{d}\right]^{-1} .
\end{aligned}
$$

Let $d^{u}$ and $d^{c}$ denote interior solutions satisfying the first-order conditions in Eqs. (1) and (2), respectively. The subscripts " $u$ " and " $c$ " mean that the choice of $d$ is "unconstrained" and "constrained" by the debt rule, respectively. When the choice is constrained, the government can break the rule by incurring additional costs. The solutions are expressed as functions of $s_{12}$ and $s_{23}$ as follows:

$$
\begin{aligned}
d^{u}\left(s_{12}, s_{23}\right) & =\frac{k-[1+\beta(1+\eta)]\left(s_{12}-s_{23}\right)}{(1+\beta)(1+\eta)}, \\
d^{c}\left(s_{12}, s_{23}\right) & =\frac{k+(\gamma+\omega) \bar{d}-[1+\beta(1+\eta+\gamma+\omega)]\left(s_{12}-s_{23}\right)}{(1+\beta)(1+\eta+\gamma+\omega)},
\end{aligned}
$$


where $d^{u}\left(s_{12}, s_{23}\right)$ and $d^{c}\left(s_{12}, s_{23}\right)$ satisfy

$d^{c}\left(s_{12}, s_{23}\right) \gtreqless \bar{d} \Leftrightarrow A\left(s_{12}, s_{23}\right) \equiv \frac{k-[1+\beta(1+\eta+\gamma+\omega)]\left(s_{12}-s_{23}\right)}{1+\eta+\beta(1+\eta+\gamma+\omega)} \gtreqless \bar{d}$,

and $^{4}$

$$
A\left(s_{12}, s_{23}\right) \leq d^{u}\left(s_{12}, s_{23}\right) .
$$

The condition of $A\left(s_{12}, s_{23}\right) \leq d^{u}\left(s_{12}, s_{23}\right)$ implies that there are four possible cases, classified according to the relative magnitude among $d^{u}\left(s_{12}, s_{23}\right)$, $A\left(s_{12}, s_{23}\right)$, and $\bar{d}$, as illustrated in Figure 2: $d^{u}\left(s_{12}, s_{23}\right) \leq 0 \leq \bar{d}$ (panel (a)), $0<d^{u}\left(s_{12}, s_{23}\right)<\bar{d}$ (panel (b)), $A\left(s_{12}, s_{23}\right) \leq \bar{d} \leq d^{u}\left(s_{12}, s_{23}\right)$ (panel (c)), and $\bar{d}<A\left(s_{12}, s_{23}\right)$ (panel $\left.(\mathrm{d})\right)$. From the figure, we find that the solution $d$ for the government problem, denoted by $d^{*}\left(s_{12}, s_{23}\right)$, as follows:

$$
d^{*}\left(s_{12}, s_{23}\right)=\left\{\begin{array}{cl}
0 & \text { when } d^{u}\left(s_{12}, s_{23}\right) \leq 0 \\
d^{u}\left(s_{12}, s_{23}\right) & \text { when } 0<d^{u}\left(s_{12}, s_{23}\right)<\bar{d} \\
\bar{d} & \text { when } A\left(s_{12}, s_{23}\right) \leq \bar{d} \leq d^{u}\left(s_{12}, s_{23}\right) \\
d^{c}\left(s_{12}, s_{23}\right) & \text { when } \bar{d}<A\left(s_{12}, s_{23}\right)
\end{array}\right.
$$

[Figure 2.]

Consider $d^{u}\left(s_{12}, s_{23}\right)$, which represents the optimal level of public debt when it satisfies the debt ceiling. Eq. (3) indicates that $d^{u}\left(s_{12}, s_{23}\right)$ increases as $\left(s_{12}-s_{23}\right)$ and $\beta$ decrease. The term $\left(s_{12}-s_{23}\right)$, representing the period- 2 consumption when there is no debt issue, implies that the marginal utility of period-2 consumption increases as $\left(s_{12}-s_{23}\right)$ decreases. The term $\beta$, representing the present bias, implies that the period- 2 selves attach a larger weight on the period- 2 consumption relative to the period- 3 consumption as $\beta$ decreases. Thus, the period- 2 selves' preferences for debt financing increase as $\left(s_{12}-s_{23}\right)$ and $\beta$ decrease.

More precisely, suppose that $\left(s_{12}-s_{23}\right)$ and $\beta$ are high, such that $d^{u}\left(s_{12}, s_{23}\right) \leq$ 0 holds. Then, the optimal level of public debt is below zero. In other words, the government prefers to lend rather than borrow in the international market. However, lending is not allowed in the present framework. Thus, the government's choice is constrained by the non-negativity constraint; the optimal level of public debt becomes $d^{*}=0$, as illustrated in panel (a) of Figure 2. When $\left(s_{12}-s_{23}\right)$ and

\footnotetext{
${ }^{4}$ Proof of $A\left(s_{12}, s_{23}\right) \leq d^{u}\left(s_{12}, s_{23}\right)$ is as follows: Suppose, contrastingly, that $A\left(s_{12}, s_{23}\right)>$ $d^{u}\left(s_{12}, s_{23}\right)$, that is, $0>k / \eta+s_{12}-s_{23}$ holds. The period-2 budget constraint leads to $c_{2} \leq$ $s_{12}+d-s_{23}<s_{12}+k / \eta-s_{23}$, where the second inequality comes from $d \leq \bar{d}<k / \eta$. Given $c_{2}>0$, this implies that $0<s_{12}+k / \eta-s_{23}$, which is a contradiction.
} 
$\beta$ are at moderate levels, such that $0<d^{u}\left(s_{12}, s_{23}\right)<\bar{d}$, the government is not constrained by the non-negativity constraint or the debt ceiling. Thus, its choice is $d^{*}=d^{u}\left(s_{12}, s_{23}\right)$, as illustrated in panel (b) of Figure 1 .

Finally, when $\left(s_{12}-s_{23}\right)$ and $\beta$ are low, such that $\bar{d} \leq d^{u}\left(s_{12}, s_{23}\right)$ holds, the government may borrow over the debt ceiling. In particular, its decision depends on the relative magnitude between $A\left(s_{12}, s_{23}\right)$ and $\bar{d}$. Since $A\left(s_{12}, s_{23}\right)$ is decreasing in $(\gamma+\omega)$, which represents the costs of rule-breaking, the government finds it is optimal to follow the rule and issues debt up to the limit, $d^{*}=\bar{d}$, when $(\gamma+\omega)$ is large, such that $A\left(s_{12}, s_{23}\right) \leq \bar{d}$, as illustrated in panel (c) of Figure 2. However, rule-breaking occurs when $(\gamma+\omega)$ is low, such that $\bar{d}<A\left(s_{12}, s_{23}\right)$, as illustrated in panel (d) of Figure 2.

\subsection{Period-2 Selves' Decision}

Next, we consider the period-2 selves' decision regarding one-period securities, $s_{23}$. The objective function of the period-2 selves is

$$
V_{2}\left(s_{12}, s_{23}, d^{e}\right) \equiv \ln \left(s_{12}+d^{e}-s_{23}\right)+\beta \ln \left[k-s_{12}+s_{23}-G\left(d^{e}\right)\right] .
$$

It should be noted that period-2 selves take $d^{e}$ as given when choosing $s_{23}$ because they are infinitesimal and thus are unable to control $d$ by choosing $s_{23}$. The firstorder condition, with respect to $s_{23}$, leads to

$$
\begin{aligned}
& s_{23}=s_{23}^{u}\left(s_{12}, d^{e}\right) \equiv s_{12}-\frac{k-(1+\eta+\beta) d^{e}}{1+\beta} \text { when } d^{e} \leq \bar{d}, \\
& s_{23}=s_{23}^{c}\left(s_{12}, d^{e}\right) \equiv s_{12}-\frac{k+(\gamma+\omega) \bar{d}-(1+\eta+\gamma+\omega+\beta) d^{e}}{1+\beta} \text { when } d^{e}>\bar{d} .
\end{aligned}
$$

With the private borrowing constraint, $s_{23} \geq 0$, and the expectation of $d=d^{e}$, an optimal level of $s_{23}$, denoted by $s_{23}^{*}$, is given by

$$
s_{23}^{*}\left(s_{12}, d^{e}\right)=\left\{\begin{array}{cl}
0 & \text { when } d^{e} \leq \bar{d} \text { and } s_{12} \leq S^{u}\left(d^{e}\right) \\
s_{23}^{u}\left(s_{12}, d^{e}\right) & \text { when } d^{e} \leq \bar{d} \text { and } s_{12}>S^{u}\left(d^{e}\right) \\
0 & \text { when } d^{e}>\bar{d} \text { and } s_{12} \leq S^{c}\left(d^{e}\right) \\
s_{23}^{c}\left(s_{12}, d^{e}\right) & \text { when } d^{e}>\bar{d} \text { and } s_{12}>S^{c}\left(d^{e}\right)
\end{array}\right.
$$


where $S^{u}\left(d^{e}\right)$ and $S^{c}\left(d^{e}\right)$, are defined as follows:

$$
\begin{aligned}
S^{u}\left(d^{e}\right) & \equiv \frac{k-(1+\eta+\beta) d^{e}}{1+\beta}, \\
S^{c}\left(d^{e}\right) & \equiv \frac{k+(\gamma+\omega) \bar{d}-(1+\eta+\gamma+\omega+\beta) d^{e}}{1+\beta} .
\end{aligned}
$$

Period-2 selves attach larger weight to period-2 consumption than the period-1 selves. This implies that the former selves are induced to increase their period-2 consumption by lowering their saving in $s_{23}$. In particular, the period-2 selves find it optimal to save nothing in $s_{23}$ when the expectation of $d^{e}$ is low and/or when the savings in one-period securities, $s_{12}$, by the period- 1 selves is low, such that either $s_{12} \leq S^{u}\left(d^{e}\right)$ or $s_{12} \leq S^{c}\left(d^{e}\right)$ holds. If this were not the case, the period-2 selves could afford to save a portion of the return from one-period securities, $s_{12}$, in $s_{23}$.

\subsection{Period-1 Selves' Decision}

Consider the period-1 agent's objective function, which is given by

$$
V_{1}\left(s_{12}, d^{e}\right) \equiv \ln \left[s_{12}+d^{e}-s_{23}^{*}\left(s_{12}, d^{e}\right)\right]+\ln \left[k-s_{12}+s_{23}^{*}\left(s_{12}, d^{e}\right)-G\left(d^{e}\right)\right]
$$

It is assumed that period- 1 and period- 2 selves have the same expectation for $d$. Given the expectation of $d=d^{e}$, the period-1 agent chooses $s_{12}$ to maximize his/her objective. Let $s_{12}^{*}$ denote the solution to the problem. The solution satisfies the following first-order condition:

$$
\begin{aligned}
\frac{\partial V_{1}\left(s_{12}, d^{e}\right)}{\partial s_{12}}= & {\left[1-\frac{\partial s_{23}^{*}\left(s_{12}, d^{e}\right)}{\partial s_{12}}\right] \cdot\left[s_{12}+d^{e}-s_{23}^{*}\left(s_{12}, d^{e}\right)\right]^{-1} } \\
& \left.-\left[1-\frac{\partial s_{23}^{*}\left(s_{12}, d^{e}\right)}{\partial s_{12}}\right] \cdot\left[k-s_{12}+s_{23}^{*}\left(s_{12}, d^{e}\right)-G\left(d^{e}\right)\right]^{-1} \leq Q, 11\right)
\end{aligned}
$$

where a strict inequality holds if $s_{12}=0$.

\subsection{Rational Expectations Equilibrium}

Having described the behavior of agents and the government, we define the rational expectations equilibrium in the framework as follows.

Definition 1: Given a debt rule $\bar{d}$, a rational expectations equilibrium is an allocation $\left(s_{12}, s_{13}, s_{23}, c_{2}, c_{3}, d\right)$, such that (i) $s_{12}=s_{12}^{*}\left(d^{e}\right)$ solves the period- 
1 agent's problem given $s_{23}$ and $d=d^{e}$; (ii) $s_{23}=s_{23}^{*}\left(s_{12}^{*}\left(d^{e}\right), d^{e}\right)$ solves period-2 agent's problem given $s_{12}$ and $d=d^{e}$; (iii) rational expectations hold, that is, the solution to the period-2 government's problem, $d$, satisfies $d^{*}\left(s_{12}^{*}\left(d^{e}\right), s_{23}^{*}\left(s_{12}^{*}\left(d^{e}\right), d^{e}\right)\right)=d^{e}$; and (iv) given $s_{12}=s_{12}^{*}\left(d^{e}\right), s_{23}=$ $s_{23}^{*}\left(s_{12}^{*}\left(d^{e}\right), d^{e}\right)$, and $d=d^{*}\left(s_{12}^{*}\left(d^{e}\right), s_{23}^{*}\left(s_{12}^{*}\left(d^{e}\right), d^{e}\right)\right)$, allocation $\left(s_{13}, c_{2}, c_{3}\right)$ is determined by the period-1, -2 , and -3 budget constraints.

[Figure 3 is here].

To characterize the equilibrium allocation, we proceed with the analysis as follows. First, we assume that period- 1 and period-2 selves have one of the following expectations of $d$ : (i) $d^{e}=0$, (ii) $d^{e}=d^{u} \in(0, \bar{d})$, (iii) $d^{e}=\bar{d}$, and (iv) $d^{e}=d^{c}(>\bar{d})$, where $d^{u}$ and $d^{c}$ denote the expectations of agents that the debt issuance is below or above the ceiling, $\bar{d}$, respectively. Given the expectation of the debt issuance, we solve for one-period securities, $s_{12}=s_{12}^{*}\left(d^{e}\right)$ and $s_{23}=s_{23}^{*}\left(s_{12}^{*}\left(d^{e}\right), d^{e}\right)$. Then we substitute these into the solution $d=d^{*}\left(s_{12}, s_{23}\right)$ for the government problem, and identify the condition in which the expectations are rational.

Let $\bar{d}_{L}(\beta)$ and $\bar{d}_{H}(\beta)\left(>\bar{d}_{L}(\beta)\right)$ denote two threshold values of the public debt:

$$
\begin{aligned}
\bar{d}_{L}(\beta) & \equiv \frac{k}{1+\eta+\beta(1+\eta+\gamma+\omega)} \\
\bar{d}_{H}(\beta) & \equiv \frac{k}{1+\eta+\beta(1+\eta)} .
\end{aligned}
$$

With the use of these two threshold values, we can present the equilibrium level of public debt, denoted by $d^{*}$, in the following proposition. The corresponding allocation of savings and consumption is presented in the Appendix A.1.

\section{Proposition 1. (Equilibrium Public Debt for a Given Debt Rule)}

(i) There exists an equilibrium in which the equilibrium debt is beyond the ceiling, $d^{*}>\bar{d}$, if $\beta \leq 1 /(1+\eta+\gamma+\omega)$ and $\bar{d}<\bar{d}_{L}(\beta)$.

(ii) There exists a continuum of equilibria in which the equilibrium debt is beyond the ceiling, $d^{*} \in(\bar{d},(k+(\gamma+\omega) \bar{d}) /(2+\eta+\gamma+\omega))$, if $\beta=1 /(1+\eta+\gamma+\omega)$ and $\bar{d}<k /(2+\eta)$.

(iii) There exists an equilibrium in which the equilibrium debt is up to the ceiling, $d^{*}=\bar{d}$, if $(a) \max \left\{k /(2+\eta), \bar{d}_{L}(\beta)\right\} \leq \bar{d} \leq \bar{d}_{H}(\beta)$ or $(b) 1 /(1+\eta+\gamma+\omega) \leq$ $\beta \leq 1 /(1+\eta)$ and $\bar{d}<k /(2+\eta)$. 
(iv) There exists an equilibrium in which the equilibrium debt is positive and below the ceiling, $0<d^{*}<\bar{d}$, if $\beta \leq 1 /(1+\eta)$ and $\bar{d}_{H}(\beta)<\bar{d}$.

(v) There exists a continuum of equilibria in which the equilibrium debt is positive and below the ceiling, $d^{*} \in(0, \min \{k /(2+\eta), \bar{d}\})$, if $\beta=1 /(1+\eta)$ and $\bar{d}>0$.

(vi) There exists an equilibrium in which the equilibrium debt is zero, $d^{*}=0$, if $\beta \geq 1 /(1+\eta)$.

Proof. See Appendix A.1.

[Figure 4 is here].

Figure 4 takes $\beta$ in the horizontal axis and $\bar{d}$ in the vertical axis, and illustrates the classification of equilibrium states according to the level of public debt. In the area P.1(i), where $\beta$ is below the threshold value, $1 /(1+\eta+\gamma+\omega)$, rule-breaking occurs in equilibrium. The area P.1(i) widens as $\omega$ decreases. This implies that the rule-breaking is more likely to occur in the uncoordinated case, $\omega=0$, than in the coordinated case, $\omega>0$.

Figure 5 illustrates how the equilibrium levels of public debt, $d^{*}$, and oneperiod securities from period 1 to period $2, s_{12}^{*}$, change in response to a change in the degree of the present bias, represented by $\beta$. As observed in Figure 5, a lower $\beta$ is associated with a higher level of $d^{*}$ and a lower level of $s_{12}^{*}$. A lower $\beta$ implies that period-2 selves are more present biased relative to period-1 selves. In other words, period-2 consumption, as planned by period-2 selves, is excessive from the period-1 selves' viewpoint. To establish control over period-2 consumption, period-1 selves reduce one-period securities, $s_{12}^{*}$, which contribute to period-2 consumption, and instead increase two-period securities, $s_{13}^{*}$. Given this behavior of period-1 selves, period-2 selves, as voters, support more public debt issues to increase their consumption in period 2.

[Figure 5 here.]

From the figures, we see that the equilibrium level of public debt substantially changes around the two threshold values of $\beta, 1 /(1+\eta)$, and $1 /(1+\eta+\gamma+\omega)$. The mechanism behind these changes are as follows: First, consider the choice of public debt around $\beta=1 /(1+\eta)$. When $\beta$ is slightly higher than the threshold, $1 /(1+\eta)$, period-2 selves have little incentive to support public debt issues via 
voting. Period-1 selves can control period-2 selves' decisions by saving decisions in period 1 and attain the first-best allocation. However, when $\beta$ is slightly lower than the threshold, $1 /(1+\eta)$, period-1 selves are unable to prevent period-2 selves from issuing public debt. This limitation induces period-1 selves to cut the savings on one-period securities, $s_{12}^{*}$. To compensate for this loss of savings, period-2 selves choose to considerably increase the public debt issue. This is the mechanism behind the substantial changes in the amount of public debt around the threshold $\beta=1 /(1+\eta)$.

Second, consider the choice of public debt around $\beta=1 /(1+\eta+\gamma+\omega)$. When $\beta$ is slightly higher than the threshold, $1 /(1+\eta+\gamma+\omega)$, period-2 selves support and choose public debt issue up to the ceiling, $d^{*}=\bar{d}$, This implies that for a $\beta$ that is slightly lower than the threshold, $1 /(1+\eta+\gamma+\omega)$, period- 2 selves have an incentive to support the public debt issue beyond the ceiling at the cost of rule-breaking. Given this expected behavior on the part of period-2 selves, period1 selves reduce the one-period securities, $s_{12}^{*}$, to control the excess consumption in period 2. Period-2 selves, in turn, choose to increase public debt issues to compensate for the loss of the return from one-period securities. Therefore, there is a substantial change in the amount of public debt around the threshold of $\beta=1 /(1+\eta+\gamma+\omega)$, when the ceiling $\bar{d}$ is below $k /(2+\eta)$. However, such change does not occur when the debt ceiling is high, such that $\bar{d}>k /(2+\eta)$ holds. In this case, the equilibrium level of one-period securities, $s_{12}^{*}$, is zero for a $\beta$ that is slightly higher than the threshold $1 /(1+\eta+\gamma+\omega)$. Thus, period- 1 selves are unable to reduce $s_{12}^{*}$ further in response to a decrease in $\beta$.

\section{Debt Rule Making}

Thus far, we assume that the government takes the debt rule, represented by $\bar{d}$, as given. This assumption - which follows Bisin, Lizzeri and Yariv (2015) - is reasonable in the short run, but in the long run, there must be a tendency toward revising debt rules, as described in the Introduction. This section extends the analysis in the previous sections by introducing endogenous determination of the debt rule via voting.

We examine two types of rules, the uncoordinated rule (in Subsection 4.1) and the coordinated rule (in Subsection 4.2), and compare them in terms of welfare, focusing on the role of present bias in the formation of the rules (in Subsection 4.3). We assume that the debt rule is determined before the period-1 selves decide on saving $s_{12}$ and $s_{13}$. Thus, the debt rule is set to maximize the indirect utility 
of period-1 selves.

\subsection{Uncoordinated Debt Rules}

We first consider the uncoordinated rule $\omega=0$. Under this rule, each country independently sets the debt ceiling to maximize the welfare of its citizens. The following proposition provides the optimal debt ceiling, the equilibrium level of debt, and the associated welfare under the uncoordinated rule.

Proposition 2. In the uncoordinated rule case, the optimal debt ceiling for the period-1 selves, $\bar{d}^{*, u c}$, is

$$
\bar{d}^{*, u c} \begin{cases}=\bar{d}_{L}(\beta) & \text { if } 0 \leq \beta<\frac{1}{1+\eta+\gamma}, \\ =\left\{\frac{k}{2+\eta}, 0\right\} & \text { if } \beta=\frac{1}{1+\eta+\gamma} \\ =0 & \text { if } \frac{1}{1+\eta+\gamma}<\beta \leq \frac{1}{1+\eta} \\ \in[0, k / \eta] & \text { if } \frac{1}{1+\eta}<\beta \leq 1 .\end{cases}
$$

The equilibrium level of debt and the associated welfare level, $d^{*, u c}(\beta)$, and $W^{*, u c}(\beta)$, are

$$
\begin{aligned}
d^{*, u c}(\beta)=\left\{\begin{array}{cl}
\bar{d}_{L}(\beta) & \text { if } 0 \leq \beta<\frac{1}{1+\eta+\gamma}, \\
\left\{\frac{k}{2+\eta}, 0\right\} & \text { if } \beta=\frac{1}{1+\eta+\gamma}, \\
0 & \text { if } \frac{1}{1+\eta+\gamma}<\beta \leq 1,
\end{array}\right. \\
W^{*, u c}(\beta)=\left\{\begin{array}{cl}
V_{(i i i, a)}^{\omega=0}\left(\bar{d}_{L}(\beta)\right) & \text { if } 0 \leq \beta<\frac{1}{1+\eta+\gamma}, \\
\left.V_{(i i i, a)}^{\omega=0}\left(\bar{d}_{L}(\beta)\right), 2 \ln \left(\frac{k}{2}\right)\right\} & \text { if } \beta=\frac{1}{1+\eta+\gamma}, \\
2 \ln \left(\frac{k}{2}\right) & \text { if } \frac{1}{1+\eta+\gamma}<\beta \leq 1,
\end{array}\right.
\end{aligned}
$$

where

$$
V_{(i i i, a)}^{\omega=0}\left(\bar{d}_{L}(\beta)\right)=\ln \left[\frac{\beta(1+\eta+\gamma) k^{2}}{\{1+\eta+\beta(1+\eta+\gamma)\}^{2}}\right]
$$

Proof. See Appendix A.2.

Figure 6 illustrates the results in Proposition 2, showing that the optimal debt ceiling depends on the degree of present bias, $\beta$. When the present bias is weak, to the extent such that $1 /(1+\eta)<\beta$ holds, period- 1 selves can curb period-2 selves' excessive consumption and the corresponding debt issues only through saving decisions on one- and two-period securities, $s_{12}$ and $s_{13}$. In other 
words, debt ceilings are irrelevant for controlling period-2 selves' behavior. Thus, period-1 selves can attain the first-best allocation regardless of the levels of debt ceilings.

[Figure 6 is here.]

When the present bias is strong, to the extent such that $\beta \leq 1 /(1+\eta)$ holds, period-1 selves cannot curb period-2 selves' behavior only through saving decisions. Debt ceilings are relevant for controlling period-2 selves' excessive consumption and public debt issues. In particular, when $\beta$ is within the range, $(1 /(1+\eta+\gamma), 1 /(1+\eta)]$, setting the debt ceiling at $\bar{d}^{*}=0$ prevents period-2 selves from choosing excess consumption and public debt. Thus, period-1 selves can attain the first-best allocation by managing savings, $s_{12}$ and $s_{13}$, and debt ceilings, $\bar{d}$.

However, when $\beta$ is given, such that $\beta<1 /(1+\eta+\gamma)$ holds, setting the debt ceiling at $\bar{d}^{*}=0$ induces period-2 selves to break the debt ceiling and thus creates additional costs for period-1 selves. To avoid such cost increases, period-1 selves need to set the debt ceiling at a maximum level such that period-2 selves never break it. This implies that period-2 selves' incentive for rule-breaking determines the standard of the rule chosen by period-1 selves. Thus, when the degree of present bias is strong, such that $\beta<1 /(1+\eta+\gamma)$ holds, period-2 selves' excessive consumption is not fully controlled by period-1 selves' decisions on saving and debt ceilings; the first-best allocation is not implementable in this case.

In terms of policy implications, our results suggest that the implementation of debt ceilings contribute to managing possible excessive consumption and the associated overissue of public debt by period-2 selves only for the case with moderate values of $\beta \in(1 /(1+\eta+\gamma), 1 /(1+\eta)]$. The effectiveness of debt ceilings is limited for the case with low values of $\beta$, to an extent such that $\beta<1 /(1+\eta+\gamma)$ holds. In this case, the possibility of rule-breaking by period-2 selves shapes period-1 selves' decisions on debt ceilings and thus induces them to adopt lax, rather than strict, rules.

\subsection{Coordinated Debt Rules}

In this section, we consider the case in which the debt rule is set in a coordinated way. In particular, we assume that all countries form a fiscal union and coordinate to set the debt ceiling that applies to members of the union. In other words, participation in the setting of the coordinated debt rule is mandatory rather than 
optional for all member countries. ${ }^{5}$ In this situation, countries are expected to have the following costs and benefits arising from their participation in the fiscal union. First, the same debt ceiling is applied to every member country, but it is generally too high or too low relative to each country's optimal ceiling. This gives the welfare losses associated with the deviation from each country's optimal debt ceiling. Second, the penalty $(\omega>0)$, applied to countries that break the coordinated debt rule, incentivizes them to control overissuance of public debt. This generates welfare improvement for the member countries, especially for those with a low value of $\beta$ such that $\beta<1 /(1+\eta+\gamma)$ holds.

To evaluate such costs and benefits arising from the fiscal coordination, we assume that countries form a fiscal union that aims to maximize a Benthamite social welfare function that is a weighted sum of the utility functions of member countries. For tractability of analysis, $\beta$ is defined as a uniform distribution from 0 to 1 . Under this assumption, the social welfare function, denoted by $W^{u}(\bar{d})$, is given as follows:

$$
W^{U}(\bar{d})= \begin{cases}W_{\text {low }}^{U}(\bar{d}) & \text { when } 0 \leq \bar{d}<\frac{k}{2+\eta} \\ W_{\text {mid }}^{U}(\bar{d}) & \text { when } \frac{k}{2+\eta} \leq \bar{d}<\frac{k}{1+\eta} \\ W^{U}(\bar{d})_{\text {high }} & \text { otherwise }\end{cases}
$$

where $W_{\text {low }}^{u}(\bar{d}), W_{\text {mid }}^{u}(\bar{d})$, and $W_{\text {high }}^{u}(\bar{d})$ denotes the social welfare function when $\bar{d}$ is below $k /(2+\eta)$, between $k /(2+\eta)$ and $k /(1+\eta)$, and above $k /(1+\eta)$, respectively. They are defined as:

$$
\begin{aligned}
W_{\text {low }}^{U}(\bar{d}) & =\int_{0}^{\frac{1}{1+\eta+\gamma+\omega}} V_{(i)}^{\omega>0}(\bar{d}) \mathrm{d} \beta+\int_{\frac{1}{1+\eta+\gamma+\omega}}^{\frac{1}{1+\eta}} V_{(i i i, b)}^{\omega>0}(\bar{d}) \mathrm{d} \beta+\int_{\frac{1}{1+\eta}}^{1} V_{(v i)}^{\omega>0} \mathrm{~d} \beta, \\
W_{\text {mid }}^{U}(\bar{d}) & =\int_{0}^{\beta_{1}(\bar{d})} V_{(i)}^{\omega>0}(\bar{d}) \mathrm{d} \beta+\int_{\beta_{1}(\bar{d})}^{\beta_{2}(\bar{d})} V_{(i i i, a)}^{\omega>0}(\bar{d}) \mathrm{d} \beta+\int_{\beta_{2}(\bar{d})}^{\frac{1}{1+\eta}} V_{(i v)}^{\omega>0} \mathrm{~d} \beta+\int_{\frac{1}{1+\eta}}^{1} V_{(v i)}^{\omega>0} \mathrm{~d} \beta, \\
W_{\text {high }}^{U} & =\int_{0}^{\frac{1}{1+\eta}} V_{(i v)}^{\omega>0} \mathrm{~d} \beta+\int_{\frac{1}{1+\eta}}^{1} V_{(v i)}^{\omega>0} \mathrm{~d} \beta,
\end{aligned}
$$

where $V_{j}^{\omega>0}(\bar{d})(j=(i),(i i i, a),(i i i, b),(i v)$, and $(v i))$ denote the indirect utility

\footnotetext{
${ }^{5}$ The optional case will be discussed later.
} 
associated with the statement $j$ in Proposition 1 and is defined as follows:

$$
\begin{aligned}
V_{(i)}^{\omega>0}(\bar{d}) & =2 \ln [k+(\gamma+\omega) \bar{d}]+\ln \left[\frac{\beta}{(1+\beta)^{2}(1+\eta+\gamma+\omega)}\right], \\
V_{(i i i, a)}^{\omega>0}(\bar{d}) & =\ln (\bar{d})+\ln [k-(1+\eta) \bar{d}] \\
V_{(i i i, b)}^{\omega>0}(\bar{d}) & =2 \ln \left(\frac{k-\eta \bar{d}}{2}\right) \\
V_{(i v)}^{\omega>0} & =\ln \left[\frac{\beta k^{2}}{(1+\beta)^{2}(1+\eta)}\right] \\
V_{(v i)}^{\omega>0} & =2 \ln \left(\frac{k}{2}\right)
\end{aligned}
$$

Here, two remarks are in order. First, $V_{(i i)}^{\omega>0}$ and $V_{(v)}^{\omega>0}$, representing the indirect utility functions at $\beta=1 /(1+\eta+\gamma+\omega)$ and $\beta=1 /(1+\eta)$, respectively, are not included in the expression in (14). This is because the equilibrium at $\beta=1 /(1+\eta+\gamma+\omega)$ and $\beta=1 /(1+\eta)$ is a measure zero set. Second, the two threshold values of $\beta, \beta_{1}(\bar{d})$ and $\beta_{2}(\bar{d})$, as shown in Eq. (16) are defined as the solutions to $\bar{d}=\bar{d}_{H}(\beta)$ and $\bar{d}=\bar{d}_{L}(\beta)$ for $\beta$, respectively. They are defined as follows:

$$
\begin{aligned}
& \bar{d}=\frac{k}{1+\eta+\beta(1+\eta+\gamma+\omega)} \Leftrightarrow \quad \beta=\frac{k-(1+\eta) \bar{d}}{(1+\eta+\gamma+\omega) \bar{d}} \equiv \beta_{1}(\bar{d}), \\
& \bar{d}=\frac{k}{(1+\beta)(1+\eta)} \quad \Leftrightarrow \quad \beta=\frac{k-(1+\eta) \bar{d}}{(1+\eta) \bar{d}} \equiv \beta_{2}(\bar{d}) .
\end{aligned}
$$

The following proposition provides the optimal debt ceiling set by the fiscal union.

Proposition 3. In the coordinated rule case, the optimal debt ceiling is

$$
\bar{d}^{*, c}=\left\{\begin{array}{l}
\frac{1}{1+\eta+\gamma+\omega} \cdot \frac{k}{\eta} \in\left(0, \frac{k}{2+\eta}\right) \text { when } 2<\eta(\eta+\gamma+\omega), \\
\frac{k\left[(\gamma+\omega)+\sqrt{(\gamma+\omega)^{2}+8(1+\eta)(1+\eta+\gamma+\omega)}\right]}{4(1+\eta)(1+\eta+\gamma+\omega)} \in\left[\frac{k}{2+\eta}, \frac{k}{1+\eta}\right) \text { otherwise. }
\end{array}\right.
$$

Proof. See Appendix A.3.

The result in Proposition 3 indicates that the optimal debt ceiling in the coordinated case, $\bar{d}^{*, c}$, is below $k /(1+\eta)$. Looking back at Figure 4 , we can see that when $\bar{d}^{*, c}<k /(1+\eta)$ holds, there are following two groups of countries: the 
first, labeled P.1(i), includes countries that break the debt ceiling, and the second, labeled P.1(iii,a) and P.1(iii,b), includes countries that choose public debt issues up to the ceiling. The fiscal union takes account of the presence of such groups of countries, and finds that setting the debt ceiling below $k /(1+\eta)$ is optimal from the viewpoint of social welfare maximization.

The intuition behind the choice of the fiscal union is as follows. First, suppose, conversely, that the fiscal union sets the debt ceiling beyond $k /(1+\eta)$. Looking back again in Figure 4, we find that in such a case, no country breaks the rule or issues public debt up to the ceiling. Under this situation, a marginal change in the debt ceiling has no effect on each country's behavior and thus, does not affect social welfare.

Next, suppose that the fiscal union sets the debt ceiling at $\bar{d}^{*, c}=k /(1+\eta)$, and consider a marginal further reduction in the debt ceiling from $k /(1+\eta)$. Then, some of the countries included in the group labeled P.1(iv) in Figure 4 moves on to the group labeled P.1(i) or P.1(iiia). A move of countries to group P.1(iii,a) contributes to social welfare improvement because the debt ceiling lowers the distortionary costs of revenue collection by public debt issues. At the same time, the move of the countries to group P.1(i) leads to social welfare losses because the debt issuance beyond the ceiling creates additional costs and inefficiencies. Around $\bar{d}^{*, c}=k /(1+\eta)$, there are two opposing effects on social welfare, and the former benefit always outweighs the latter cost in the present framework. Thus, a further marginal reduction of the debt ceiling from $\bar{d}^{*, c}=k /(1+\eta)$ leads to social welfare improvement. This in turn incentivizes the fiscal union to set the ceiling below $\bar{d}^{*, c}=k /(1+\eta)$. Therefore, there are always countries that issue public debt up to or beyond the debt ceiling.

\subsection{Coordination and Present Bias}

In this subsection, we compare the uncoordinated and coordinated cases in terms of debt levels and social welfare, focusing on the role of present bias in the formation of the debt rule. We assume that the cost of debt issues and rule-breaking are high such that the following condition holds.

Assumption 2. $2<\eta(\eta+\gamma+\omega)$.

Assumption 2 allows us to focus on the first case presented in Proposition 3. The result presented below does not change substantially if the second case in Proposition 3 is considered (see Appendix B.) 
We first compare the uncoordinated and coordinated cases in terms of debt levels. The debt level in the uncoordinated case is provided in Proposition 2. The debt level in the coordinated case is derived as follows. Recall that under Assumption 2, the debt ceiling in the coordinated case is

$$
\bar{d}^{*, c}=\frac{1}{1+\eta+\gamma+\omega} \cdot \frac{k}{\eta} \in\left(0, \frac{k}{2+\eta}\right) .
$$

Given this debt ceiling, there are five groups of countries, labeled P.1(i), P.1(ii), P.1(iii,b), P.1(v), and P.1(vi), as observed in Figure 4. The associated level of debt for each group, denoted by $d^{*, c}$, is

$$
d^{*, c}(\beta) \begin{cases}=\frac{(1+\eta)(\eta+\gamma+\omega)}{(1+\beta)(1+\eta+\gamma+\omega)^{2}} \cdot \frac{k}{\eta} & \text { when } 0 \leq \beta \leq \frac{1}{1+\eta+\gamma+\omega}, \\ \in\left(\frac{1}{1+\eta+\gamma+\omega} \cdot \frac{k}{\eta}, \frac{(1+\eta)(\eta+\gamma+\omega)}{(2+\eta+\gamma+\omega)(1+\eta+\gamma+\omega)} \cdot \frac{k}{\eta}\right) & \text { when } \beta=\frac{1}{1+\eta+\gamma+\omega}, \\ \in\left(0, \frac{1}{1+\eta+\gamma+\omega} \cdot \frac{k}{\eta}\right. & \text { when } \frac{1}{1+\eta+\gamma+\omega} \leq \beta \leq \frac{1}{1+\eta}, \\ =0 & \text { when } \beta=\frac{1}{1+\eta}, \\ & \text { when } \beta \geq \frac{1}{1+\eta} .\end{cases}
$$

We write $d^{*, c}$ as a function of $\beta$ to emphasize the role of the present bias in the process of public debt accumulation.

Proposition 4. Suppose that Assumptions 1 and 2 hold. The debt level is (i) lower (higher) in the coordinated rule case than in the uncoordinated rule case if $0 \leq \beta<1 /(1+\eta+\gamma)(1 /(1+\eta+\gamma) \leq \beta<1 /(1+\eta))$; and (ii) equal between the two cases if $1 /(1+\eta)<\beta \leq 1$ :

$$
d^{*, c}(\beta) \begin{cases}<d^{*, u c}(\beta) & \text { if } 0 \leq \beta<\frac{1}{1+\eta+\gamma}, \\ >d^{*, u c}(\beta) & \text { if } \frac{1}{1+\eta+\gamma}<\beta<\frac{1}{1+\eta}, \\ =d^{*, u c}(\beta)=0 & \text { if } \frac{1}{1+\eta}<\beta \leq 1 .\end{cases}
$$

Proof. See Appendix A.4.

Figure 7 illustrates the result of Proposition 4, taking $\beta$ on the horizontal axis. Consider the group of countries with high values of $\beta$ to the extent such that 
$\beta \in(1 /(1+\eta), 1]$ holds. These countries can attain the first-best allocation is distinguished by no public debt issue regardless of whether the rule is coordinated or uncoordinated. This is because a high value of $\beta$ implies that period- 2 selves are weakly biased toward current consumption. This, in turn, implies that they have no incentive to spend today more than they planned before by issuing public debt. Thus, the public debt issue is zero for any given level of the debt ceiling when $\beta$ is high such that $\beta \in(1 /(1+\eta), 1]$ holds.

[Figure 7 here.]

Next, consider the group of countries with $\beta \in[0,1 /(1+\eta)]$. This group is divided into the two sub-groups and includes (i) countries with low values of $\beta$ to the extent such that $\beta \in[0,1 /(1+\eta+\gamma)]$ holds; and (ii) countries with moderate values of $\beta$ such that $\beta \in[1 /(1+\eta+\gamma), 1 /(1+\eta))$ holds. As for the former group of countries, the public debt issuance is limited in the coordinated case relative to the uncoordinated case. However, the opposite result holds for the latter.

To understand the mechanism of this variation, we consider the former group of countries. Period-2 selves in these countries are heavily biased toward the current (i.e., period-2) consumption. The period-2 selves would support the issue of public debt beyond the debt ceiling if this is set low. In the uncoordinated case, the period-1 selves consider this rule-breaking possibility and set the debt ceiling through voting at a maximum level such that the period-2 selves never break it. In the coordinated case, the period-2 selves are more restrained in supporting the public debt issue than in the uncoordinated case. This is because there are additional costs for rule-breaking. presented by $\omega$ in the coordinated case. This means that the period-2 selves may follow a coordinated debt rule even if it is stricter than that in the uncoordinated case. Therefore, the countries concerned here can restrain the amount of public debt to be issued by participating in the international coordination of the debt ceiling.

Consider the latter group of countries, such that $\beta \in[1 /(1+\eta+\gamma), 1 /(1+\eta)]$ holds. These countries attain the first-best allocation characterized by no public debt issue in the uncoordinated case. Period-2 selves have an incentive to issue public debt to finance their consumption today (i.e., in period 2). However, the period-1 selves fully suppress this incentive by setting the debt ceiling to zero. Such full control is not realized in the coordinated case because some amount of debt is allowed to be issued in this case. Thus, the period-1 selves in these countries are made worse off by participating in the international coordination of the debt ceiling. 
Next, compare the coordinated and uncoordinated rule cases in terms of social welfare. The social welfare in the uncoordinated rule case is provided in Proposition 2. The following lemma provides the social welfare in the coordinated rule case.

Lemma 1. Suppose that Assumptions 1 and 2 hold. The social welfare in the coordinated rule case, denoted by $V^{*, c}(\beta)$, is

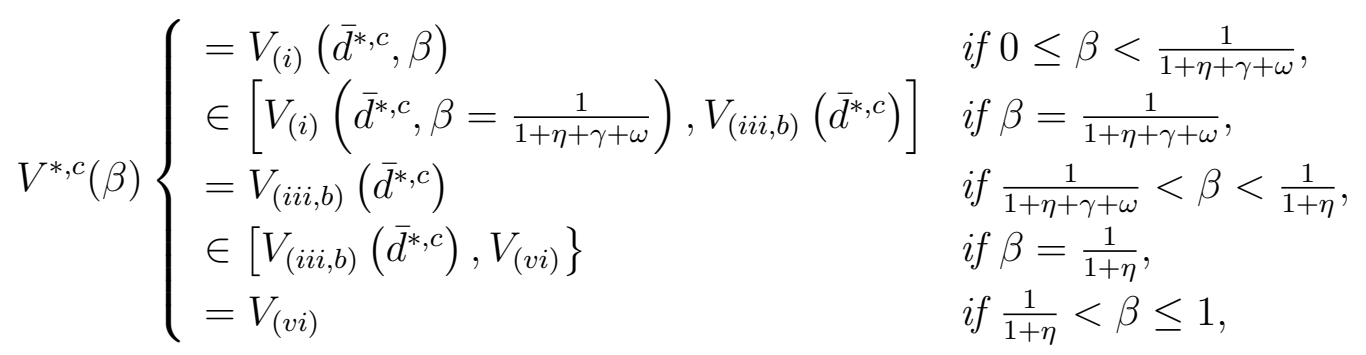

where $V_{(i)}^{\omega>0}\left(\bar{d}^{*, c}, \beta\right), V_{(i i i, b)}^{\omega>0}\left(\bar{d}^{*, c}, \beta\right)$, and $V_{(v i)}^{\omega>0}\left(\bar{d}^{*, c}, \beta\right)$ are defined by

$$
\begin{aligned}
V_{(i)}\left(\bar{d}^{*, c}, \beta\right) & =2 \ln \left[\frac{(1+\eta)(\eta+\gamma+\omega)}{1+\eta+\gamma+\omega} \cdot \frac{k}{\eta}\right]+\ln \left[\frac{\beta}{(1+\beta)^{2}(1+\eta+\gamma+\omega)}\right] \\
V_{(i i i, b)}\left(\bar{d}^{*, c}\right) & =2 \ln \left[\frac{\eta(\eta+\gamma+\omega)}{2(1+\eta+\gamma+\omega)} \cdot \frac{k}{\eta}\right] \\
V_{(v i)} & =2 \ln \left(\frac{k}{2}\right) .
\end{aligned}
$$

Proof. See Appendix A.5.

Figure 8 takes $\beta$ on the horizontal axis and illustrates the social welfare in the coordinated rule case. The following three points are noted from Fig. 8. First, consider countries populated by agents with weak present bias such that $\beta>1 /(1+\eta)$ holds. Given a weak present bias of the period-2 selves, period1 selves can curb period-2 selves' excessive consumption and the associated debt issue only through saving decisions on one- and two-period securities, $s_{12}$, and $s_{13}$. The debt ceiling is irrelevant for controlling period-2 selves' behavior. Therefore, the period-1 selves can attain the first-best allocation, and the associated social welfare is provided at the highest level.

[Figure 8 here.]

Second, consider countries populated by agents with moderate present bias such that $1 /(1+\eta)<\beta<1 /(1+\eta+\gamma+\omega)$ holds. Period-1 selves cannot fully control period-2 selves' excessive consumption and the corresponding debt issue 
only through savings. In other words, the period-2 selves consume more than is optimal from the period-1 selves' perspective. However, the period-2 selves' incentive for excessive consumption can be controlled in part by the coordinated debt ceiling. Therefore, the debt levels are bounded at $\bar{d}^{*, c}$ for the countries concerned here, and these countries attain the same level of social welfare.

Finally, consider countries populated by agents with strong bias such that $\beta<1 /(1+\eta+\gamma+\omega)$ holds. In these countries, the first-best allocation is not realized as in the former case. In addition, the period-2 selves choose to issue public debt beyond the coordinated debt ceiling level. This incentive of overissue of debt becomes strengthened by the present bias, and in turn, increases rulebreaking costs. Therefore, social welfare decreases as $\beta$ decreases.

Based on the results established in Proposition 2 and Lemma 1, we compare the coordinated and uncoordinated rule cases in terms of social welfare.

Proposition 5. Suppose that Assumptions 1 and 2 hold. The following relation holds between social welfare in the coordinated rule case, $W^{*, c}(\beta)$, and that in the uncoordinated rule case, $W^{*, u c}(\beta)$.

(i) When $\beta>1 /(1+\eta+\gamma+\omega)$ holds,

$$
W^{*, c}(\beta)\left\{\begin{array}{l}
>W^{*, u c}(\beta) \quad \text { if } \frac{1}{1+\eta+\gamma+\omega}<\beta<\frac{1}{1+\eta+\gamma} \\
<W^{*, u c}(\beta) \text { if } \frac{1}{1+\eta+\gamma}<\beta<\frac{1}{1+\eta} \\
=W^{*, u c}(\beta) \text { if } \frac{1}{1+\eta}<\beta \leq 1
\end{array}\right.
$$

(ii) When $\beta<1 /(1+\eta+\gamma+\omega)$ holds,

$$
W^{*, c}(\beta) \begin{cases}\leq W^{*, u c}(\beta) \text { if } \quad & (i) \phi_{L} \geq 0, \text { or } \\ & (\text { ii }) \phi_{H}>0>\phi_{L} \text { and }(a) \frac{1}{1+\eta+\gamma+\omega} \leq-\frac{\phi_{H}}{\phi_{L}}, \text { or }(b) \beta \leq-\frac{\phi_{H}}{\phi_{L}}<\frac{1}{1+\eta+\gamma+\omega}, \\ >W^{*, u c}(\beta) \text { if } \quad & (\text { iii }) \phi_{H} \leq 0, \text { or } \\ & (\text { iv }) \phi_{H}>0>\phi_{L} \text { and }-\frac{\phi_{H}}{\phi_{L}}<\beta<\frac{1}{1+\eta+\gamma+\omega},\end{cases}
$$

where $\phi_{L}$ and $\phi_{H}$ are defined by

$$
\begin{aligned}
\phi_{L} & \equiv(1+\eta+\gamma+\omega) \eta \sqrt{(1+\eta+\gamma)(1+\eta+\gamma+\omega)}-(1+\eta)(1+\eta+\gamma)(\eta+\gamma+\omega) \\
\phi_{H} & \equiv(1+\eta+\gamma+\omega) \eta \sqrt{(1+\eta+\gamma)(1+\eta+\gamma+\omega)}-(1+\eta)^{2}(\eta+\gamma+\omega)>\phi_{L} .
\end{aligned}
$$

Proof. See Appendix A.6. 
Figure 9 takes $\beta$ on the horizontal axis, and plots $W^{*, c}(\beta)$ and $W^{*, u c}(\beta)$ to show how they change as $\beta$ varies from 0 to 1 . As shown in Proposition 5 , some countries benefit from the coordination, while others lose. The costs and benefits of participating in coordination depend on the three effects, namely unifying, penalty, and distortionary effects. Next, we define these effects and describe how these effects work in our framework.

[Figure 9.]

The unifying effect is the effect that occurs when the same debt rule, which would not be optimal for each member of the fiscal union, is applied to all countries. Countries with large (small) values of $\beta$ would suffer from welfare losses associated with less (more) strict debt rule in the coordinated rule case relative to the uncoordinated rule case. The penalty effect stems from the presence of rule breaking costs in the coordinated rule case, denoted by $\omega(>0)$. The costs may produce welfare improvement associated with a disincentive for each country to break the debt ceiling. However, some countries with small values of $\beta$ might break the coordinated debt rule even in the presence of such rule-breaking costs because people in these countries are sufficiently impatient. This results in the welfare losses arising from the rule-breaking: this is called the distortionary effect.

The three effects may or may not occur, or occur to varying degrees, depending on the present bias, $\beta$. First, suppose that $\beta$ is high to the extent such that $\beta>1 /(1+\eta)$ holds. Period-2 selves living in such a high- $\beta$ country are sufficiently patient, so period-1 selves can control period-2 selves' decisions only through period-1 saving. They can attain the first-best allocation with no debt issue irrespective of the rule chosen.

Second, suppose that $\beta$ is slightly lower relative to the one considered in the first case such that $1 /(1+\eta+\gamma)<\beta<1 /(1+\eta)$ holds. Period-2 selves are less patient relative to the first case. In the uncoordinated rule case, period-1 selves set the debt ceiling at zero because they cannot fully curb period-2 selves' behavior through saving decisions. In the coordinated rule case, all countries share the same debt ceiling that allows for a positive debt issue. This produces welfare losses associated with the unifying effect that gives an incentive for the period-2 selves to issue public debt up to the limit. Thus, social welfare is lower in the coordinated rule case than in the uncoordinated rule case. It should be noted that penalty and distortionary effects do not work in the current case as $\beta$ is not as small as the effects appear. 
Third, suppose that $\beta$ is lower than for the previous two cases such that $1 /(1+\eta+\gamma+\omega)<\beta<1 /(1+\eta+\gamma)$ holds. As described in Proposition 2 below, in the uncoordinated rule case, setting the debt ceiling at the first-best level, $\bar{d}^{*}=0$, induces the period-2 selves to break the debt ceiling. To control the rule-breaking incentive, period- 1 selves set the debt ceiling at a minimum level such that period- 2 selves would never break it. In the coordinated rule case, there is no incentive for rule-breaking because of the presence of the penalty effect. Period-2 selves follow the coordinated debt rule and issue public debt up to the limit. Ultimately, period-2 selves follow the debt rule and issue public debt up to the limit in both cases. However, social welfare is higher in the coordinated rule case than in the uncoordinated rule case because the debt ceiling is set at a lower level in the coordinated rule case than in the uncoordinated rule case. The discrepancy in the debt ceilings between the two cases comes from the unifying effect observed in the coordinated rule case.

Finally, suppose that $\beta$ is sufficiently low such that $\beta<1 /(1+\eta+\gamma+\omega)$ holds. The period- 2 selves living in such a low- $\beta$ country are sufficiently impatient, and thus have a strong incentive to cover the consumption needs in period 2 by issuing public debt. Given such an incentive, the debt ceiling is set at a lower level in the coordinated rule case than in the uncoordinated rule case: this is the unifying effect that works to lower social welfare. At the same time, coordination lowers social welfare because period-2 selves choose to issue public debt beyond the debt ceiling, which creates the distortionary effect. However, the penalty effect, providing a disincentive for each country to break the dent ceiling, curbs the overissuance of public debt and leads to social welfare improvements. Which effect dominates depends on the relative magnitude between the three effects. Figure 9 illustrates three cases which may be observed in (a) and (b) with $\beta \leq-\phi_{h} / \phi_{L}$, the sum of the unifying and distortionary effects dominates the penalty effect, resulting in social welfare losses; and in (b) with $\beta>-\phi_{h} / \phi_{L}$ and (c), the penalty effect dominates the sum of the unifying and distortionary effects, resulting in social welfare improvement.

The last case of $\beta<1 /(1+\eta+\gamma+\omega)$ is presented in Proposition 5(ii); the case implies that the welfare consequence of the coordination depends on the structural parameters including $\beta, \eta, \gamma$, and $\omega$, but their effects are not clearly identified from the conditions presented in 5(ii). To resolve this problem and evaluate the effects of the parameters more accurately, we use numerical simulations. Figure 10 depicts a set of $(\gamma, \omega)$ that satisfies the conditions in Proposition 5(ii) for three cases: (a) $\eta=0.5$, (b) $\eta=1.0$, and (c) $\eta=1.5$. 
[Figure 10 is here].

The following observations are made from Figure 10. First, when $\omega$ is sufficiently high, either of the following conditions holds: $\phi_{L} \geq 0$, or $-\phi_{H} / \phi_{L} \geq$ $1 /(1+\eta+\gamma+\omega)$. The distortionary effect is strengthened, and thus social welfare is reduced as the rule-breaking costs under coordination, $\omega$, increases. Thus, the coordination of the debt rules results in social welfare losses when $\omega$ is sufficiently high.

Second, when $\omega$ is low, the social welfare consequence of coordination depends on $\gamma$, representing the rule-breaking costs relevant for both cases. The distortionary effect becomes stronger as the rule breaking costs, $\gamma$, increases. Thus, social welfare is reduced by coordination when $\gamma$ is high. However, when $\gamma$ is low, the unifying and distortionary effects are outweighed by the penalty effect, and social welfare is improved by coordination when $\gamma$ is low.

Finally, suppose that $\omega$ is low but $\gamma$ is moderate such that $0<-\phi_{H} / \phi_{L}<$ $1 /(1+\eta+\gamma+\omega)$ holds. In this case, the relative magnitude between the penalty, unifying, and distortionary effects depends on the present bias, $\beta$. The distortionary effect is strengthened as the present bias increases (that is, as $\beta$ decreases). The unifying and distortionary effects outweighs the penalty effect and thus coordination lowers social welfare if $\beta$ is low; the opposite result holds if $\beta$ is high.

\subsection{Policy Implications}

Propositions 2 to 5 provide the following policy implications: First, propositions 2 to 4 lead to the consequence that the introduction of the coordinated rule has the effect of reducing the volume of bond issuance in countries with strongly present-biased preferences and excessive debt (see Figure 7). In other words, the coordinated rule is more effective than the uncoordinated rule in achieving the objective of curbing the excessive issuance of public debt and restoring fiscal health. However, if participation in the coordinated rule is optional for each country, then, as Proposition 5 suggests, whether countries are willing to adopt the coordinated rule depends on the relative size of the three effects mentioned above (see Figure 9).

In considering the three effects, we focus on the case where the unifying and distortionary effects dominate the penalty effect. In this case, countries facing strongly present-biased preferences and excessive bond issuance suffer from welfare losses as a result of participating in the coordinated rule (see Figure 9 (a) and (b)). This result suggests that such countries will refuse to participate in 
the coordinated rule. The results also suggest that countries that are already participating in the coordinated rule are willing to withdraw from it. This, in turn, implies that the possibility of deviating from the coordinated rule makes it difficult to introduce and maintain the coordinated rule that contributes to the fiscal health of regional economies such as the EU. Therefore, to get countries to commit to the coordinated rule, it will be necessary to implement measures such as the acceptance of standards violations in times of economic downturn or broader cooperation among countries on fiscal issues.

\section{Conclusion}

Households with present-biased preferences have the incentive to consume more in the present compared to their previous consumption plans, which they previously considered optimal. When such households choose a fiscal policy through voting, they choose a policy of increasing bond issuance. To control the excessive issuance of government bonds through household voting behavior, it is necessary to introduce rules to cap the issuance of government bonds. The model of Bisin, Lizzeri, and Yariv (2015), which focuses on the role of such debt rules, is extended in this study to an economy consisting of a large number of countries with varying degrees of present bias and the possibility of debt rule violation. Within this extended framework, this study evaluated the effectiveness of coordinated debt rules, such as those introduced in the EU, by conducting a comparative analysis of the case where each country sets its own rules versus a common set of fiscal rules for all countries within an economic area.

We show that whether a tighter debt ceiling is achieved under a coordinated or uncoordinated rule depends on the degree of present bias in each country. In the case of coordinated rules, the rules are based on the average present bias of all countries, but in the case of uncoordinated rules, each rule is based on the degree of present bias of each country. Thus, our analysis implies that the ceiling on government bond issuance under the coordinated rule is tighter for countries with strong present-biased preferences and looser for countries with weak presentbiased preferences.

The transition from uncoordinated rules to coordinated rules has an impact on government bond issuance and welfare in each country. For countries with strong present-biased preferences, participation in the coordinated rule would lead to a tighter ceiling, which would reduce the volume of government bond issuance. By contrast, for countries with weak present-biased preferences, participation in the 
coordinated rule loosens the ceiling and thus increasing government bond issuance. Our analysis indicates that a uniform imposition of coordinated fiscal rules on all countries is not necessarily desirable, since the impacts of rule changes on the volume of government debt issuance and social welfare of each country depend on its degree of present bias and thus vary from country to country. Therefore, our results suggest that the degree of present bias in each country should be considered when implementing internationally coordinated debt rules. 


\section{A Proofs}

\section{A.1 Proof of Proposition 1 and the Equilibrium Allocation}

In the following, we show seven propositions, labeled $\mathrm{Ai}(\mathrm{i}=1,2,3,4,5,6,7)$. They correspond, in order, to results (i), (ii), (iiia), and (iiib), (iv), (v), and (vi) in Proposition 1.

\section{A.1.1 Equilibrium with $d>\bar{d}$}

Suppose that period-1 and period-2 selves expect that $d^{e}=d^{c}(>\bar{d})$ holds. Eq. (8) leads to the savings in period-2 selves, when $d^{e}=d^{c}(>\bar{d})$ as follows:

$$
s_{23}^{*}\left(s_{12}, d^{c}\right)=\left\{\begin{array}{cl}
0 & \text { when } s_{12} \leq S^{c}\left(d^{c}\right) \\
s_{23}^{c}\left(s_{12}, d^{c}\right) & \text { when } s_{12}>S^{c}\left(d^{c}\right) .
\end{array}\right.
$$

Figure A.1 illustrates $V_{1}\left(s_{12}, d^{c}\right)$. When $s_{12} \leq S^{c}\left(d^{c}\right)$ holds, the first-order condition, with respect to $s_{12}$ in (11), is rewritten as follows:

$$
\frac{\partial V_{1}\left(s_{12}, d^{c}\right)}{\partial s_{12}}=\left(s_{12}+d^{c}\right)^{-1}-\left[k-s_{12}-(1+\eta+\gamma+\omega) d^{c}+(\gamma+\omega) \bar{d}\right]^{-1} \leq 0,
$$

where an interior solution is given by

$$
s_{12}=\frac{k+(\gamma+\omega) \bar{d}-(2+\eta+\gamma+\omega) d^{c}}{2}
$$

[Figure A.1 here.]

Alternatively, when $s_{12}>S^{c}\left(d^{c}\right)$ holds, the first-order condition with respect to $s_{12}$ in (11), becomes

$$
\frac{\partial V_{1}\left(s_{12}, d^{c}\right)}{\partial s_{12}}=0
$$

suggesting that $V_{1}$ is independent of $s_{12}$ as long as $s_{12}>S^{c}\left(d^{c}\right)$ holds. Notice that $V_{1}\left(s_{12}, d^{c}\right)$ is continuous at $s_{12}=S^{c}\left(d^{c}\right)$, as illustrated in Figure A.1.

The interior solution of $s_{12}$ and the threshold value $S^{c}\left(d^{c}\right)$ are compared as follows:

$$
S^{c}\left(d^{c}\right) \gtreqless \frac{k+(\gamma+\omega) \bar{d}-(2+\eta+\gamma+\omega) d^{c}}{2} \Leftrightarrow d^{c} \lesseqgtr \frac{k+(\gamma+\omega) \bar{d}}{\eta+\gamma+\omega} .
$$


In addition, the following conditions hold:

$$
\begin{aligned}
\frac{k+(\gamma+\omega) \bar{d}-(2+\eta+\gamma+\omega) d^{c}}{2} \gtreqless 0 \Leftrightarrow d^{c} \lesseqgtr \frac{k+(\gamma+\omega) \bar{d}}{2+\eta+\gamma+\omega}, \\
S^{c}\left(d^{c}\right) \gtreqless 0 \Leftrightarrow d^{c} \lesseqgtr \frac{k+(\gamma+\omega) \bar{d}}{1+\eta+\gamma+\omega+\beta} .
\end{aligned}
$$

Furthermore, the three threshold values of $d^{c}$ are ranked as

$$
\frac{k+(\gamma+\omega) \bar{d}}{2+\eta+\gamma+\omega}<\frac{k+(\gamma+\omega) \bar{d}}{1+\eta+\gamma+\omega+\beta}<\frac{k+(\gamma+\omega) \bar{d}}{\eta+\gamma+\omega}
$$

Given these properties, we can conclude that the optimal levels of $s_{12}$ and $s_{23}$ when $d^{e}=d^{c}(>\bar{d})$, are given as follows:

(i) $s_{12}^{*} \in[0, k], s_{23}^{*}=s_{12}-\frac{k+(\gamma+\omega) \bar{d}-(1+\eta+\gamma+\omega+\beta) d^{c}}{1+\beta}$

$$
\begin{gathered}
s_{12}^{*}=0, s_{23}^{*}=0 \\
s_{12}^{*}=\frac{k+(\gamma+\omega) \bar{d}-(2+\eta+\gamma+\omega) d^{c}}{2}, s_{23}^{*}=0
\end{gathered}
$$

when $\frac{k+(\gamma+\omega) \bar{d}}{1+\eta+\gamma+\omega+\beta} \leq d^{c}$ when $\frac{k+(\gamma+\omega) \bar{d}}{2+\eta+\gamma+\omega} \leq d^{c}<\frac{k+(\gamma+\omega) \bar{d}}{1+\eta+\gamma+\omega+\beta}$, when $d^{c}<\frac{k+(\gamma+\omega) \bar{d}}{2+\eta+\gamma+\omega}$.

Next, we determine the conditions, such that the expectation of $d^{e}=d^{c}(>\bar{d})$ is rational, for the three cases in (A.1).

Case of $(k+(\gamma+\omega) \bar{d}) /(1+\eta+\gamma+\omega+\beta) \leq d^{c}$

From (4) and (A.1), the expectation of $d^{e}=d^{c}$ is rational if the following condition holds:

$$
d^{c}=\frac{k+(\gamma+\omega) \bar{d}-[1+\beta(1+\eta+\gamma+\omega)] \cdot \frac{k+(\gamma+\omega) \bar{d}-(1+\eta+\gamma+\omega+\beta) d^{c}}{1+\beta}}{(1+\beta)(1+\eta+\gamma+\omega)}
$$

and $(k+(\gamma+\omega) \bar{d}) /(1+\eta+\gamma+\omega+\beta) \leq d^{c}$, or

$$
\frac{k+(\gamma+\omega) \bar{d}}{1+\eta+\gamma+\omega+\beta} \leq d^{c}=\frac{k+(\gamma+\omega) \bar{d}}{\eta+\gamma+\omega}
$$

The associated level of $s_{23}$ is

$$
s_{23}^{*}=s_{12}^{*}+\frac{k+(\gamma+\omega) \bar{d}}{\eta+\gamma+\omega},
$$

and the corresponding consumption levels are $c_{2}=c_{3}=0$, which contradicts the first-order conditions with respect to $c_{2}$ and $c_{3}$. Thus, there is no rational expectations equilibrium in this case. 
Case of $(k+(\gamma+\omega) \bar{d}) /(2+\eta+\gamma+\omega) \leq d^{c}<(k+(\gamma+\omega) \bar{d}) /(1+\eta+\gamma+\omega+\beta)$

From (4) and (A.1), the expectation of $d^{e}=d^{c}$ is rational if the following conditions hold:

$$
d^{c}=\frac{k+(\gamma+\omega) \bar{d}}{(1+\beta)(1+\eta+\gamma+\omega)} \text { and } \frac{k+(\gamma+\omega) \bar{d}}{2+\eta+\gamma+\omega} \leq d^{c}<\frac{k+(\gamma+\omega) \bar{d}}{1+\eta+\gamma+\omega+\beta} .
$$

This level of public debt is above the limit, $\bar{d}$, if

$$
\bar{d}<\frac{k+(\gamma+\omega) \bar{d}}{(1+\beta)(1+\eta+\gamma+\omega)} \Leftrightarrow \bar{d}<\frac{k}{1+\eta+\beta(1+\eta+\gamma+\omega)}=\bar{d}_{L}(\beta) .
$$

In addition, $d^{c}$ must satisfy the second condition in (A.2):

$$
\frac{k+(\gamma+\omega) \bar{d}}{2+\eta+\gamma+\omega} \leq d^{c}=\frac{k+(\gamma+\omega) \bar{d}}{(1+\beta)(1+\eta+\gamma+\omega)}<\frac{k+(\gamma+\omega) \bar{d}}{1+\eta+\gamma+\omega+\beta}
$$

The first inequality holds if and only if $\beta \leq 1 /(1+\eta+\gamma+\omega)$, whereas the second inequality always holds.

Proposition A.1. Suppose that $\beta \leq \frac{1}{1+\eta+\gamma+\omega}$ and $\bar{d}<\bar{d}_{L}(\beta)$ hold. There is a rational expectations equilibrium with $d=\frac{k+(\gamma+\omega) \bar{d}}{(1+\beta)(1+\eta+\gamma+\omega)} \in\left(\bar{d}, \frac{k}{\eta}\right)$, and

$$
\left(c_{2}, c_{3}, s_{12}, s_{13}, s_{23}\right)=\left(\frac{k+(\gamma+\omega) \bar{d}}{(1+\beta)(1+\eta+\gamma+\omega)}, \frac{\beta(k+(\gamma+\omega) \bar{d})}{1+\beta}, 0, k, 0\right) .
$$

Case of $d^{c}<(k+(\gamma+\omega) \bar{d}) /(2+\eta+\gamma+\omega)$

From (4) and (A.1), the expectation of $d^{e}=d^{c}$ is rational if the following conditions hold:

$$
\begin{gathered}
{[1-\beta(1+\eta+\gamma+\omega)] \cdot\left[(k+(\gamma+\omega) \bar{d})-(\eta \cdot+\gamma+\omega) d^{c}\right]=0} \\
\text { and } d^{c}<\frac{k+(\gamma+\omega) \bar{d}}{2+\eta+\gamma+\omega} .
\end{gathered}
$$

The first condition in (A.3) indicates that the rational expectation of public debt is given by:

$$
d^{c}\left\{\begin{array}{cl}
\in\left(\bar{d}, \frac{k}{\eta}\right) & \text { if } \beta=\frac{1}{1+\eta+\gamma+\omega} \\
=\frac{k+(\gamma+\omega) \bar{d}}{\eta+\gamma+\omega} & \text { if } \beta \neq \frac{1}{1+\eta+\gamma+\omega}
\end{array}\right.
$$


When $\beta \neq 1 /(1+\eta+\gamma+\omega), d^{c}=(k+(\gamma+\omega) \bar{d}) /(\eta+\gamma+\omega)$ must satisfy the second condition in (A.3):

$$
d^{c}=\frac{k+(\gamma+\omega) \bar{d}}{\eta+\gamma+\omega}<\frac{k+(\gamma+\omega) \bar{d}}{2+\eta+\gamma+\omega}
$$

however, this inequality condition fails to hold. Alternatively, when $\beta=1 /(1+\eta+\gamma+\omega)$, $d^{c} \in(\bar{d}, k / \eta)$ with the second condition in (A.3) gives the equilibrium level for rational expectation of public debt as

$$
d \in\left(\bar{d}, \frac{k+(\gamma+\omega) \bar{d}}{2+\eta+\gamma+\omega}\right)
$$

where the set is nonempty if $\bar{d}<k /(2+\eta)$.

Proposition A.2. Suppose that $\beta=\frac{1}{1+\eta+\gamma+\omega}$ and $\bar{d}<\frac{k}{2+\eta}$ hold. There is a rational expectations equilibrium with $d \in\left(\bar{d}, \frac{k+(\gamma+\omega) \bar{d}}{2+\eta+\gamma+\omega},\right)$ and

$$
\begin{aligned}
\left(c_{2}, c_{3}, s_{12}, s_{13}, s_{23}\right) & =\left(\frac{k+(\gamma+\omega) \bar{d}-(\eta+\gamma+\omega) d}{2}, \frac{k+(\gamma+\omega) \bar{d}-(\eta+\gamma+\omega) d}{2},\right. \\
& \left.\frac{k+(\gamma+\omega) \bar{d}-(2+\eta+\gamma+\omega) d}{2}, \frac{k-(\gamma+\omega) \bar{d}+(2+\eta+\gamma+\omega) d}{2}, 0\right) .
\end{aligned}
$$

\section{A.1.2 Equilibrium with $d=\bar{d}$}

Suppose that period-1 and period-2 selves expect that $d^{e}=\bar{d}$ holds. Equation (8) leads to the period-2 selves' saving when $d^{e}=\bar{d}$ as follows:

$$
s_{23}^{*}\left(s_{12}, \bar{d}\right)=\left\{\begin{array}{cl}
0 & \text { when } s_{12} \leq S^{u}(\bar{d}), \\
s_{12}-\frac{k-(1+\eta+\beta) \cdot \bar{d}}{1+\beta} & \text { when } s_{12}>S^{u}(\bar{d}) .
\end{array}\right.
$$

Panel (a) of Figure A.2 illustrates $V_{1}\left(s_{12}, \bar{d}\right)$. Using the same procedure as in Section A.1.1, we can conclude that the optimal levels of $s_{12}$ and $s_{23}$, when $d^{e}=\bar{d}$, become:

(i) $s_{12}^{*} \in[0, k], s_{23}^{*}=s_{12}-\frac{k-(1+\eta+\beta) \bar{d}}{1+\beta}$ when $\frac{k}{1+\eta+\beta} \leq \bar{d}$,

(ii) $\quad s_{12}^{*}=0, s_{23}^{*}=0 \quad$ when $\frac{k}{2+\eta} \leq \bar{d}<\frac{k}{1+\eta+\beta}$,

(iii) $\quad s_{12}^{*}=\frac{k-(2+\eta) \bar{d}}{2}, s_{23}^{*}=0 \quad$ when $\bar{d}<\frac{k}{2+\eta}$.

[Figure A.2 here.] 
Next, we determine the conditions, such that the expectation of $d^{e}=\bar{d}$ is rational, for the three cases in (A.4).

Case of $k /(1+\eta+\beta) \leq \bar{d}$

From (5) and (A.4), the expectation of $d^{e}=\bar{d}$ is rational if the following condition holds:

$$
\begin{aligned}
& A\left(s_{12}^{*} \in[0, k], s_{23}^{*}=s_{12}^{*}-\frac{k-(1+\eta+\beta) \bar{d}}{1+\beta}\right) \leq \bar{d} \\
& \quad \leq d^{u}\left(s_{12}^{*} \in[0, k], s_{23}^{*}=s_{12}^{*}-\frac{k-(1+\eta+\beta) \bar{d}}{1+\beta}\right) \text { and } \frac{k}{1+\eta+\beta} \leq \bar{d}
\end{aligned}
$$

The inequality $\bar{d} \leq d^{u}(\cdot, \cdot)$ is rewritten as

$$
\bar{d} \leq \frac{k-[1+\beta(1+\eta)] \frac{k-(1+\eta+\beta) \bar{d}}{1+\beta}}{(1+\beta)(1+\eta)} \Leftrightarrow \eta \leq 0,
$$

which fails to hold for any $\eta>0$. Thus, there is no rational expectations equilibrium with $d=\bar{d}$ when $k /(1+\eta+\beta) \leq \bar{d}$.

Case of $k /(2+\eta) \leq \bar{d}<k /(1+\eta+\beta)$

From (5) and (A.4), the expectation of $d^{e}=\bar{d}$ is rational if the following condition holds:

$$
A\left(s_{12}^{*}=0, s_{23}^{*}=0\right) \leq \bar{d} \leq d^{u}\left(s_{12}^{*}=0, s_{23}^{*}=0\right) \quad \text { and } \frac{k}{2+\eta} \leq \bar{d}<\frac{k}{1+\eta+\beta},
$$

that is, if

$$
\begin{aligned}
\bar{d}_{L}(\beta)=\frac{k}{1+\eta+\beta(1+\eta+\gamma+\omega)} & \leq \bar{d} \leq \frac{k}{1+\eta+\beta(1+\eta)}=\bar{d}_{H}(\beta) \text { and } \\
\frac{k}{2+\eta} & \leq \bar{d}<\frac{k}{1+\eta+\beta} .
\end{aligned}
$$

These are summarized as in the following propositions:

Proposition A.3. Suppose that $\max \left\{\frac{k}{2+\eta}, \bar{d}_{L}(\beta)\right\} \leq \bar{d} \leq \bar{d}_{H}(\beta)$ holds. There is a rational expectations equilibrium with $d=\bar{d}$ and

$$
\left(c_{2}, c_{3}, s_{12}, s_{13}, s_{23}\right)=(\bar{d}, k-(1+\eta) \bar{d}, 0, k, 0) .
$$


Case of $\bar{d}<k /(2+\eta)$

From (5) and (A.4), the expectation of $d^{e}=\bar{d}$ is rational if the following conditions hold:

$$
\begin{aligned}
A\left(s_{12}^{*}\right. & \left.=\frac{k-(2+\eta) \bar{d}}{2}, s_{23}^{*}=0\right) \leq \bar{d} \\
& \leq d^{u}\left(s_{12}^{*}=\frac{k-(2+\eta) \bar{d}}{2}, s_{23}^{*}=0\right) \text { and } \bar{d}<\frac{k}{2+\eta} .
\end{aligned}
$$

The first condition in (A.5) is reformulated as follows:

$$
\frac{k-[1+\beta(1+\eta+\gamma+\omega)] \frac{k-(2+\eta) \bar{d}}{2}}{1+\eta+\beta(1+\eta+\gamma+\omega)} \leq \bar{d} \Leftrightarrow \frac{1}{1+\eta+\gamma+\omega} \leq \beta,
$$

and

$$
\bar{d} \leq \frac{k-[1+\beta(1+\eta)] \frac{k-(2+\eta) \bar{d}}{2}}{(1+\beta)(1+\eta)} \Leftrightarrow \beta \leq \frac{1}{1+\eta} .
$$

The equilibrium conditions are summarized in the following proposition.

Proposition A.4. Suppose that $\frac{1}{1+\eta+\gamma+\omega} \leq \beta \leq \frac{1}{1+\eta}$ and $\bar{d}<\frac{k}{2+\eta}$ hold. There is a rational expectations equilibrium with $d=\bar{d}$ and

$$
\left(c_{2}, c_{3}, s_{12}, s_{13}, s_{23}\right)=\left(\frac{k-\eta \bar{d}}{2}, \frac{k-\eta \bar{d}}{2}, \frac{k-(2+\eta) \bar{d}}{2}, \frac{k+(2+\eta) \bar{d}}{2}, 0\right) .
$$

\section{A.1.3 Equilibrium with $d \in(0, \bar{d})$}

Suppose that period-1 and period-2 selves expect that $d^{e}=d^{u} \in(0, \bar{d})$ holds. Eq. (8) reduces by period-2 selves when $d^{e}=d^{u} \in(0, \bar{d})$ as follows:

$$
s_{23}^{*}\left(s_{12}, d^{u}\right)=\left\{\begin{array}{cl}
0 & \text { when } s_{12} \leq S^{u}\left(d^{u}\right) \\
s_{23}^{u}\left(s_{12}, d^{u}\right) & \text { when } s_{12}>S^{u}\left(d^{u}\right) .
\end{array}\right.
$$

Panel (b) of Figure A.2 illustrates $V_{1}\left(s_{12}, d^{u}\right)$. Using the same procedure as in Section A.1.1, we can conclude that the optimal levels of $s_{12}$ and $s_{23}$, when $d^{e}=d^{u}$, become

(i) $s_{12}^{*} \in[0, k], s_{23}^{*}=s_{12}-\frac{k-(1+\eta+\beta) d^{u}}{1+\beta}$ when $\frac{k}{1+\eta+\beta} \leq d^{u}$,

(ii) $\quad s_{12}^{*}=0, s_{23}^{*}=0 \quad$ when $\frac{k}{2+\eta} \leq d^{u}<\frac{k}{1+\eta+\beta}$,

(iii) $\quad s_{12}^{*}=\frac{k-(2+\eta) d^{u}}{2}, s_{23}^{*}=0$ when $d^{u}<\frac{k}{2+\eta}$. 
Next, we determine the conditions such that the expectation of $d^{e}=d^{u}$ is rational for the three cases in (A.6).

Case of $k /(1+\eta+\beta) \leq d^{u}$

From (3) and (A.6), the expectation of $d^{e}=d^{u}$ is rational if the following conditions hold:

$$
d^{u}=\frac{k-[1+\beta(1+\eta)] \cdot \frac{k-(1+\eta+\beta) \cdot d^{u}}{1+\beta}}{(1+\beta)(1+\eta)} \text { and } \frac{k}{1+\eta+\beta} \leq d^{u} .
$$

Solving the first condition in (A.7) for $d^{u}$ leads to $d^{u}=k / \eta$. Following the same reasoning as in the previous case, this candidate is not suitable for the solution. Thus, there is no rational expectations equilibrium with $k /(1+\eta+\beta) \leq d$.

Case of $k /(2+\eta) \leq d^{u}<k /(1+\eta+\beta)$

From (3) and (A.6), the expectation of $d^{e}=d^{u}$ is rational if the following conditions hold:

$$
d^{u}=\frac{k}{1+\eta+\beta(1+\eta)}=\bar{d}_{H}(\beta) \text { and } \frac{k}{2+\eta} \leq d^{u}<\frac{k}{1+\eta+\beta},
$$

that is,

$$
\frac{k}{2+\eta} \leq \bar{d}_{H}(\beta)<\frac{k}{1+\eta+\beta} .
$$

The first inequality holds if and only if $\beta(1+\eta) \leq 1$; the second inequality always holds. In addition, $d^{u}$ must satisfy $d^{u}<\bar{d}$, that is,

$$
d^{u}=\frac{k}{1+\eta+\beta(1+\eta)}=\bar{d}_{H}(\beta)<\bar{d}
$$

Proposition A.5. Suppose that $\beta \leq \frac{1}{1+\eta}$ and $\bar{d}_{H}(\beta)<\bar{d}$ hold. There is a rational expectations equilibrium with $d=\bar{d}_{H}(\beta)$ and

$$
\left(c_{2}, c_{3}, s_{12}, s_{13}, s_{23}\right)=\left(\frac{k}{1+\eta+\beta(1+\eta)}, \frac{\beta k}{1+\beta}, 0, k, 0\right) .
$$


Case of $d^{u}<k /(2+\eta)$

From (3) and (A.6), the expectation of $d^{e}=d^{u}$ is rational if the following conditions hold:

$$
[1-\beta(1+\eta)] \cdot \eta d^{u}=[1-\beta(1+\eta)] \cdot k \text { and } d^{u}<\frac{k}{2+\eta}
$$

The first condition in (A.8) implies that the rational expectations level of $d^{u}$ is given by

$$
d^{u}\left\{\begin{array}{cc}
\in(0, \bar{d}) & \text { when } \beta(1+\eta)=1 \\
=k / \eta & \text { when } \beta(1+\eta) \neq 1 .
\end{array}\right.
$$

When $\beta(1+\eta) \neq 1$, the candidate for the solution is $d^{u}=k / \eta$. This candidate is not suitable for the solution because a focus on the case of $d<\bar{d}$ and $\bar{d}<k / \eta$ is assumed in Assumption 1. When $\beta(1+\eta)=1$, any level of $d^{u} \in(0, \bar{d})$ with $d^{u}<k /(2+\eta)$ is rational. Thus, the equilibrium level of public debt becomes

$$
d \in\left(0, \min \left\{\frac{k}{2+\eta}, \bar{d}\right\}\right)
$$

where the set is nonempty if $\bar{d}>0$.

Proposition A.6. Suppose that $\beta(1+\eta)=1$ and $\bar{d}>0$ hold. There is a rational expectations equilibrium with $d \in\left(0, \min \left\{\frac{k}{2+\eta}, \bar{d}\right\}\right)$ and

$$
\left(c_{2}, c_{3}, s_{12}, s_{13}, s_{23}\right)=\left(\frac{k-\eta d}{2}, \frac{k-\eta d}{2}, \frac{k-(2+\eta) d}{2}, \frac{k+(2+\eta) d}{2}, 0\right) .
$$

\section{A.1.4 Equilibrium with $d=0$}

Suppose that period-1 and period-2 selves expect that $d^{e}=0$ holds. Eq. (8) leads to saving by period-2 selves when $d^{e}=0$ as follows:

$$
s_{23}^{*}\left(s_{12}, 0\right)=\left\{\begin{array}{cl}
0 & \text { when } s_{12} \leq S^{u}(0), \\
s_{23}^{u}\left(s_{12}, 0\right) \equiv s_{12}-\frac{k}{1+\beta} & \text { when } s_{12}>S^{u}(0) .
\end{array}\right.
$$

Panel (c) of Figure A.2 illustrates $V_{1}\left(s_{12}, 0\right)$. When $s_{12} \leq S^{u}(0)$ holds, the first-order condition, with respect to $s_{12}$ in (11), is rewritten as follows:

$$
\frac{\partial V_{1}\left(s_{12}, 0\right)}{\partial s_{12}}=\left(s_{12}\right)^{-1}-\left(k-s_{12}\right)^{-1} \leq 0
$$


An interior solution, given by $s_{12}=k / 2$, is feasible because it holds that $s_{12}=$ $k / 2<S^{u}(0) \equiv k /(1+\beta)$. Thus, an optimal level of $s_{12}$ is $s_{12}=k / 2$ when $s_{12} \leq S^{u}(0)$, as illustrated in panel (a) of Figure A.2.

Alternatively, when $s_{12}>S^{u}(0)$ holds, the first-order condition, with respect to $s_{12}$ in (11), becomes

$$
\frac{\partial V_{1}\left(s_{12}, 0\right)}{\partial s_{12}}=0
$$

suggesting that $V_{1}$ is independent of $s_{12}$ as long as $s_{12}>S^{u}(0)$ (see panel (a) of Figure A.2). Note that $V_{1}$ is continuous at $s_{12}=S^{u}(0)$.

Given the expectation of $d^{e}=0$, the optimal level of $s_{12}$ becomes

$$
s_{12}^{*}(0)=\frac{k}{2}
$$

and the corresponding level of $s_{23}$ is $s_{23}^{*}(k / 2,0)=0$. From (5), the expectation of $d^{e}=0$ is rational if the following condition holds:

$$
d^{u}\left(s_{12}^{*}, s_{23}^{*}\right)=d^{u}\left(\frac{k}{2}, 0\right) \leq 0 \Leftrightarrow \beta \geq \frac{1}{1+\eta}
$$

Proposition A.7. Suppose that $\beta \geq \frac{1}{1+\eta}$ holds. There is a rational expectations equilibrium with $d=0$ and

$$
\left(c_{2}, c_{3}, s_{12}, s_{13}, s_{23}\right)=\left(\frac{k}{2}, \frac{k}{2}, \frac{k}{2}, \frac{k}{2}, 0\right)
$$

\section{A.2 Proof of Proposition 2}

Case of $\beta<1 /(1+\eta+\gamma)$

When $\beta<1 /(1+\eta+\gamma)$, the equilibrium allocation of consumption for a given $\bar{d}$ is

$$
\left(c_{2}, c_{3}\right)=\left\{\begin{array}{cl}
\left(\frac{k+\gamma \bar{d}}{(1+\beta)(1+\eta+\gamma)}, \frac{\beta(k+\gamma \bar{d})}{1+\beta}\right) & \text { when } 0 \leq \bar{d}<\bar{d}_{L}(\beta) \\
(\bar{d}, k-(1+\eta) \bar{d}) & \text { when } \bar{d}_{L}(\beta) \leq \bar{d}<\bar{d}_{H}(\beta) \\
\left(\frac{k}{(1+\beta)(1+\eta)}, \frac{\beta k}{1+\beta}\right) & \text { when } \bar{d}_{H}(\beta) \leq \bar{d}
\end{array}\right.
$$


where the allocations come from Propositions A.1, A.3, and A.5, respectively. Thus, the period-1 selves' indirect utility function, $V_{1}(\bar{d})$, becomes:

$$
V_{1}(\bar{d})= \begin{cases}V_{(i)}^{\omega=0}(\bar{d}) & \text { when } 0 \leq \bar{d}<\bar{d}_{L}(\beta) \\ V_{(i i i, a)}^{\omega=0}(\bar{d}) & \text { when } \bar{d}_{L}(\beta) \leq \bar{d}<\bar{d}_{H}(\beta) \\ V_{(i v)}^{\omega=0} & \text { when } \bar{d}_{L}(\beta) \leq \bar{d}\end{cases}
$$

where $V_{(i)}^{\omega=0}(\bar{d}), V_{(i i i, a)}^{\omega=0}(\bar{d})$, and $V_{(i v)}^{\omega=0}(\bar{d})$, are defined by

$$
\begin{aligned}
V_{(i)}^{\omega=0}(\bar{d}) & =2 \ln (k+\gamma \bar{d})+\ln \left[\frac{\beta}{(1+\beta)^{2}(1+\eta+\gamma)}\right], \\
V_{(i i i, a)}^{\omega=0}(\bar{d}) & =\ln (\bar{d})+\ln [k-(1+\eta) \bar{d}] \\
V_{(i v)}^{\omega=0} & =\ln \left[\frac{\beta k^{2}}{(1+\beta)^{2}(1+\eta)}\right]
\end{aligned}
$$

where the subscripts (i), (iii,a), and (iv) correspond to the statements in Proposition 1 ; the superscript $\omega=0$ indicates the uncoordinated debt rule case.

The function $V_{1}(\bar{d})$ is continuous for $\bar{d} \in(0, \infty)$ because the following properties hold:

$$
\begin{aligned}
& \lim _{\bar{d} \rightarrow \bar{d}_{L}(\beta)} V_{(i)}^{\omega=0}(\bar{d})=V_{(i i i, a)}^{\omega=0}\left(\bar{d}_{L}(\beta)\right)=\ln \left[\frac{\beta(1+\eta+\gamma) k^{2}}{\{1+\eta+\beta(1+\eta+\gamma)\}^{2}}\right], \\
& \lim _{\bar{d} \rightarrow \bar{d}_{H}(\beta)} V_{(i i i, a)}^{\omega=0}(\bar{d})=V_{(i v)}^{\omega=0}=\ln \left[\frac{\beta k^{2}}{(1+\beta)^{2}(1+\eta)}\right] .
\end{aligned}
$$

In addition, the differentiation of $V_{n}^{\omega=0}(n=(i),(i i i, a),(i v))$ with respect to $\bar{d}$ leads to

$$
\begin{aligned}
\frac{\partial V_{(i)}^{\omega=0}(\bar{d})}{\partial \bar{d}} & =\frac{2 \gamma}{k+\gamma \bar{d}}>0, \\
\frac{\partial V_{(i i i, a)}^{\omega=0}(\bar{d})}{\partial \bar{d}} & =(\bar{d})^{-1}-(1+\eta)[k-(1+\eta) \bar{d}]^{-1}, \\
\frac{\partial V_{(i v)}^{\omega=0}}{\partial \bar{d}} & =0
\end{aligned}
$$

where the following condition holds:

$$
\frac{\partial V_{(i i i, a)}^{\omega=0}(\bar{d})}{\partial \bar{d}} \gtrless 0 \Leftrightarrow \bar{d} \lessgtr \frac{k}{2(1+\eta)} .
$$


Given the assumption of $\beta<1 /(1+\eta+\gamma)$, we have

$$
\frac{k}{2(1+\eta)}<\bar{d}_{L}(\beta)
$$

implying that $V_{(i i i, a)}^{\omega=0}$ is decreasing in $\bar{d}$ for the range of $\left[\bar{d}_{L}(\beta), \bar{d}_{H}(\beta)\right)$. Thus, the optimal $\bar{d}$ becomes

$$
\bar{d}^{*, u c}=\bar{d}_{L}(\beta),
$$

and the corresponding allocation of saving, consumption, and public debt is given by

$$
\left(s_{12}, s_{13}, c_{2}, c_{3}\right)=\left(0,0, \frac{k}{1+\eta+\beta(1+\eta+\gamma)}, \frac{\beta(1+\eta+\gamma) k}{1+\eta+\beta(1+\eta+\gamma)}\right) .
$$

In this case, the equilibrium debt issue and the maximized welfare under the optimal debt ceiling are given by $d^{*, u c}=\bar{d}^{*, u c}=\bar{d}_{L}(\beta)$ and

$$
W^{*, u c}=V_{(i i i, a)}^{\omega=0}\left(\bar{d}_{L}(\beta)\right)=\ln \left[\frac{\beta(1+\eta+\gamma) k^{2}}{\{1+\eta+\beta(1+\eta+\gamma)\}^{2}}\right]
$$

\section{Case of $\beta=1 /(1+\eta+\gamma)$}

From Propositions A.1, A.2, A.3, A.4, A.5, and Figure 4, period-1 selves' indirect utility function, $V_{1}(\bar{d})$, becomes

$$
V_{1}(\bar{d})=\left\{\begin{array}{cl}
\left\{V_{(i)}^{\omega=0}(\bar{d}), V_{(i i)}^{\omega=0}(\bar{d}), V_{(i i i, b)}^{\omega=0}(\bar{d})\right\} & \text { when } 0 \leq \bar{d}<\frac{k}{2+\eta} \\
V_{(i i i, a)}^{\omega=0}(\bar{d}) & \text { when } \frac{k}{2+\eta} \leq \bar{d} \leq \frac{k}{1+\eta+\frac{1+\eta}{1+\eta+\gamma}} \\
V_{(i v)}^{\omega=0} & \text { when } \frac{k}{1+\eta+\frac{1+\eta}{1+\eta+\gamma}}<\bar{d}
\end{array}\right.
$$

where $V_{(i)}^{\omega=0}(\bar{d}), V_{(i i i, a)}^{\omega=0}(\bar{d})$, and $V_{(i v)}^{\omega=0}(\bar{d})$ are given in equations (A.10) to (A.12), respectively, and $V_{(i i)}^{\omega=0}(\bar{d})$ is given by

$$
\begin{aligned}
V_{(i i)}^{\omega=0} & \equiv 2 \ln \left[\frac{k+\gamma \bar{d}-(\eta+\gamma) d}{2}\right], d \in\left(\bar{d}, \frac{k+\gamma \bar{d}}{2+\eta+\gamma}\right), \\
V_{(i i i, b)}^{\omega=0} & \equiv 2 \ln \left(\frac{k-\eta \bar{d}}{2}\right) .
\end{aligned}
$$

The subscripts (ii) in (A.17) and (iii,b) in (A.18) correspond to the statements in Proposition 1. 
To find the optimal $\bar{d}$, we first consider the maximization of $V_{1}(\bar{d})$ within the range of $\bar{d} \in[k /(2+\eta), \infty)$. The properties in (A.14), (A.15), and (A.16) indicate that $V_{(i i i, a)}^{\omega=0}(\bar{d})$ is decreasing in $\bar{d}$ for $\bar{d} \in[k /(2+\eta), \infty)$, and that $V_{(i v)}^{\omega=0}$ is independent of $\bar{d}$. Thus, $V_{1}(\bar{d})$ is maximized at $\bar{d}=k /(2+\eta)$ for the range of $\bar{d} \geq k /(2+\eta)$. The maximized value of $V_{1}(\bar{d})$ is

$$
\left.\max V_{1}(\bar{d})\right|_{\bar{d} \geq k /(2+\eta)}=V_{(i i i, a)}^{\omega=0}\left(\frac{k}{2+\eta}\right)=2 \ln \left(\frac{k}{2+\eta}\right) .
$$

Next, consider the maximization of $V_{1}(\bar{d})$ within the range $\bar{d} \in[0, k /(2+\eta))$. Recall that there are three equilibria, described in Proposition 1(i), (ii), and (iiib). Thus, the optimal $\bar{d}$ depends on the period-1 selves' expectations about the realization of the equilibrium.

\section{A.2.1 Case of Equilibrium in Proposition 1 (i)}

Suppose that the period-1 selves expect that the allocation described in Proposition 1 (i) and Proposition A.1 is realized in equilibrium. From Eq. (A.10), the period-1 selves' indirect utility function is given by

$$
V_{(i)}^{\omega=0}(\bar{d})=2 \ln (k+\gamma \bar{d})-2 \ln (2+\eta+\gamma)
$$

for $\bar{d} \in[0, k /(2+\eta))$. Because $V_{(i)}^{\omega=0}(\bar{d})$ is strictly increasing in $\bar{d}$, we obtain the supremum of $V_{(i)}^{\omega=0}(\bar{d})$ as follows:

$$
\sup _{0 \leq \bar{d}<k /(2+\eta)} V_{(i)}^{\omega=0}(\bar{d})=\lim _{\bar{d} \rightarrow k /(2+\eta)} V_{(i)}(\bar{d})=2 \ln \left(\frac{k}{2+\eta}\right)=V_{(i i i, a)}^{\omega=0}\left(\frac{k}{2+\eta}\right) .
$$

This implies that for any $\bar{d} \in[0, k /(2+\eta))$, it holdsthat that $V_{(i)}^{\omega=0}(\bar{d})<V_{(i i i, a)}^{\omega=0}(k /(2+\eta))$. Thus, the optimal $\bar{d}$ is $\bar{d}^{*, u c}=k /(2+\eta)$, when the period-1 selves expect that the equilibrium described in Proposition 1 (i) is realized.

\section{A.2.2 Case of Equilibrium in Proposition 1 (ii)}

Suppose that the period-1 selves expect that the allocation described in Proposition 1 (ii) and Proposition A.2 is realized in equilibrium. As stated in Proposition 1 (ii), there are continuum of equilibrium debt levels, $\bar{d} \in[0, k /(2+\eta))$. Next, we show that $V_{(i i)}^{\omega=0}(\bar{d})<V_{(i i i, a)}^{\omega=0}(k /(2+\eta))$ holds for any $\bar{d} \in[0, k /(2+\eta))$.

Assume that period-1 and period-2 selves form the same expectations about the debt level in period 2, and that their expectations are independent of $\bar{d}$ set 
by the period-1 government. Under this assumption, $V_{(i i)}^{\omega=0}(\bar{d})$ in Eq. (A.17) is strictly increasing in $\bar{d}$. Thus, the upper limit of $V_{(i i)}^{\omega=0}(\bar{d})$ becomes

$$
\sup _{0 \leq \bar{d}<k /(2+\eta)} V_{(i i)}^{\omega=0}(\bar{d})=\lim _{\bar{d} \rightarrow k /(2+\eta)} V_{(i i)}(\bar{d})=2 \ln \left[\frac{k+\frac{\gamma}{2+\eta} k-(\eta+\gamma) d}{2}\right] .
$$

Recall that $\inf d \rightarrow k /(2+\eta)$ when $\bar{d} \rightarrow k /(2+\eta)$. Given this property, we have

$$
\sup _{0 \leq \bar{d}<k /(2+\eta)} V_{(i i)}^{\omega=0}(\bar{d})<2 \ln \left[\frac{k+\frac{\gamma}{2+\eta} k-(\eta+\gamma) d}{2}\right]=V_{(i i i, a)}^{\omega=0}\left(\frac{k}{2+\eta}\right) .
$$

Thus, for any $\bar{d} \in[0, k /(2+\eta))$, we obtain $V_{(i i)}^{\omega=0}(\bar{d})<V_{(i i i, a)}^{\omega=0}(k /(2+\eta))$, implying that the optimal debt ceiling is $\bar{d}^{*, u c}=k /(2+\eta)$.

\section{A.2.3 Case of Equilibrium in Proposition 1 (iiib)}

Suppose that the period-1 selves expect that the allocation described in Proposition 1 (iiib) and Proposition A.4 is realized in equilibrium. The period-1 selves' indirect utility function is given by $V_{(i i i, b)}^{\omega=0}(\bar{d})$ in Eq. (A.18) for $\bar{d} \in[0, k /(2+\eta)$,$) .$ Given that $V_{(i i i, b)}^{\omega=0}(\bar{d})$ is decreasing in $\bar{d}$, we obtain

$$
\max _{0 \leq \bar{d}<k /(2+\eta)} V_{(i i i, b)}^{\omega=0}(\bar{d})=V_{(i i i, b)}^{\omega=0}(0)=2 \ln \left(\frac{k}{2}\right)>V_{(i i i, a)}^{\omega=0}\left(\frac{k}{2+\eta}\right)=2 \ln \left(\frac{k}{2+\eta}\right) .
$$

The inequality implies that the optimal $\bar{d}$ is $\bar{d}^{*, u c}=0$.

Case of $1 /(1+\eta+\gamma)<\beta \leq 1 /(1+\eta)$

Suppose that the ceiling is set at $\bar{d}=0$. Then, the allocation of consumption is

$$
\left(c_{2}, c_{3}\right)=\left(\frac{k}{2}, \frac{k}{2}\right)
$$

where the allocation comes from Proposition A.4. This allocation of consumption is consistent with the solution to the following period-1 selves' utility maximization problem:

$$
\max \ln \left(c_{2}\right)+\ln \left(c_{3}\right) \text { s.t. } c_{2}+c_{3} \leq k \text {. }
$$

Thus, the optimal level of $\bar{d}$ becomes $\bar{d}^{*, u c}=0$. 
In this case, the equilibrium debt issue and maximized welfare under the optimal debt ceiling are given by $d^{*, u c}=\bar{d}^{*, u c}=0$ and $W^{*, u c}=V_{(i i i, b)}^{\omega=0}(0)=2 \ln (k / 2)$.

Case of $\beta>1 /(1+\eta)$

When $\beta>1 /(1+\eta)$, the equilibrium allocation of consumption is

$$
\left(c_{2}, c_{3}\right)=\left(\frac{k}{2}, \frac{k}{2}\right) \quad \forall \bar{d} \in\left[0, \frac{k}{\eta}\right)
$$

where the allocation comes from Proposition A.7. Since the allocation is independent from $\bar{d}$, the optimal level of $\bar{d}$ becomes $\bar{d}^{*, u c} \in[0, k / \eta)$.

In this case, the equilibrium debt issue and the maximized welfare under the optimal debt ceiling are given by $d^{*, u c}=0$ and $W^{*, u c}=2 \ln (k / 2)$.

\section{A.3 Proof of Proposition 3}

The strategy of the proof is as follows. First, we give in Lemma A.1 six properties of the social welfare function $W^{u}(\bar{d})$ in Eq. (14). Second, we illustrate $W^{U}(\bar{d})$, taking $\bar{d}$ in the horizontal axis. Finally, with the use of the illustration of $W^{u}(\bar{d})$, we find out the optimal $\bar{d}$ that maximizes the social welfare.

Lemma A.1. The social welfare function, $W^{u}(\bar{d})$ in Eq. (14), has the following six properties:

(i) $W_{\text {low }}^{U}(\cdot)$ is strictly concave with respect to $\bar{d}$;

(ii) $\lim _{\bar{d} \rightarrow \frac{k}{2+\eta}} \frac{\partial W_{\text {low }}^{U}(\bar{d})}{\partial \bar{d}}=\left.\frac{\partial W_{m i d}^{U}(\bar{d})}{\partial \bar{d}}\right|_{\bar{d}=\frac{k}{2+\eta}} ;$

(iii) $W^{u}(\cdot)$ is continuous for $\bar{d} \in[0, k / \eta)$;

(iv) $W^{u}(\cdot)$ is strictly concave with respect to $\bar{d}$ for $\bar{d}<k /(1+\eta)$;

(v) $\lim _{\bar{d} \rightarrow \frac{k}{2+\eta}} \frac{\partial W_{\text {low }}^{U}(\bar{d})}{\partial \bar{d}}=\left.\frac{\partial W_{m i d}^{U}(\bar{d})}{\partial \bar{d}}\right|_{\bar{d}=\frac{k}{2+\eta}} \gtreqless 0 \Leftrightarrow 2 \gtreqless \eta(\eta+\gamma+\omega)$;

(vi) $\left.\frac{\partial W_{m i d}^{U}(\bar{d})}{\partial \bar{d}}\right|_{\bar{d}=\frac{k}{1+\eta}}<0$. 


\section{Proof of statement (i)}

From Eqs. (15), (18), (20), and (22), the first and second derivatives of $W_{\text {low }}^{U}(\bar{d})$ with respect to $\bar{d}$ are:

$$
\begin{aligned}
\frac{\partial W_{l o w}^{U}(\bar{d})}{\partial \bar{d}} & =\frac{2(\gamma+\omega)}{k+(\gamma+\omega) \bar{d}} \cdot \frac{1}{1+\eta+\gamma+\omega}-\frac{2 \eta}{k-\eta \bar{d}} \cdot\left[\frac{1}{1+\eta}-\frac{1}{1+\eta+\gamma+\omega}\right] \\
\frac{\partial^{2} W_{l o w}^{U}(\bar{d})}{\partial \bar{d}^{2}} & =-\frac{2(\gamma+\omega)^{2}}{[k+(\gamma+\omega) \bar{d}]^{2}} \cdot \frac{1}{1+\eta+\gamma+\omega}-\frac{2 \eta^{2}}{(k-\eta \bar{d})^{2}} \cdot\left[\frac{1}{1+\eta}-\frac{1}{1+\eta+\gamma+\omega}\right]<0 .
\end{aligned}
$$

The second derivative shows that $W_{\text {low }}^{U}(\bar{d})$ is strictly concave with respect to $\bar{d}$.

\section{Proof of statement (ii)}

From Eqs. (16), (18), (19), (21), and (22), the first derivative of $W_{m i d}^{U}(\bar{d})$ with respect to $\bar{d}$ is:

$$
\frac{\partial W_{m i d}^{U}(\bar{d})}{\partial \bar{d}}=\frac{2(\gamma+\omega)}{k+(\gamma+\omega) \bar{d}} \cdot \beta_{1}(\bar{d})+\frac{k-2(1+\eta) \bar{d}}{\bar{d}[k-(1+\eta) \bar{d}]} \cdot\left[\beta_{2}(\bar{d})-\beta_{1}(\bar{d})\right]
$$

Then, from Eqs. (23), (24), (A.21) and (A.22), we obtain

$$
\lim _{\bar{d} \rightarrow \frac{k}{2+\eta}} \frac{\partial W_{l o w}^{U}(\bar{d})}{\partial \bar{d}}=\left.\frac{\partial W_{m i d}^{U}}{\partial \bar{d}}\right|_{\bar{d}=\frac{k}{2+\eta}},
$$

showing that the slope of $W_{\text {low }}^{U}(\bar{d})$ at $\bar{d}=\frac{k}{2+\eta}$ is equivalent to the slope of $W_{\text {mid }}^{U}(\bar{d})$ at $\bar{d}=\frac{k}{2+\eta}$.

\section{Proof of statement (iii)}

First, we show that $W_{\text {low }}^{u}(\bar{d})$ and $W_{\text {mid }}^{u}(\bar{d})$ are continuous at $\bar{d}=k /(2+\eta)$. To show this, notice that the third terms on the right-hand side of Eq. (16) is zero because $\beta_{2}(k /(1+\eta))=1 /(1+\eta)$ holds from Eq. (24). In addition, the second term in Eq. (15) and the second term in Eq. (16) at $\bar{d}=k /(2+\eta)$ are reduced to:

$$
\ln (\bar{d})+\ln [k-(1+\eta) \bar{d}]=2 \ln \left(\frac{k-\eta \bar{d}}{2}\right)=2 \ln \left(\frac{k}{2+\eta}\right)
$$


Therefore, we obtain

$$
\lim _{\bar{d} \rightarrow \frac{k}{2+\eta}} W_{l o w}^{U}(\bar{d})=W_{m i d}^{U}\left(\frac{k}{2+\eta}\right)
$$

showing that $W_{\text {low }}^{u}(\bar{d})$ and $W_{\text {mid }}^{u}(\bar{d})$ are continuous at $\bar{d}=k /(2+\eta)$.

Next, we show that $W_{m i d}^{U}(\bar{d})$ and $W_{\text {high }}^{U}(\bar{d})$ are continuous at $\bar{d}=k /(1+\eta)$. From (16), (17), (23), and (24), we have

$$
\lim _{\bar{d} \rightarrow \frac{k}{1+\eta}} W_{m i d}^{U}(\bar{d})=W_{h i g h}^{U}\left(\frac{k}{1+\eta}\right)
$$

showing that $W_{\text {mid }}^{U}(\bar{d})$ and $W_{\text {high }}^{U}(\bar{d})$ are continuous at $\bar{d}=k /(1+\eta)$.

\section{Proof of statement (iv)}

With the use of Eqs. (23), (24) and (A.22), we can write down the second derivative of $W_{m i d}^{U}(\bar{d})$ with respect to $\bar{d}$ as follows:

$$
\begin{aligned}
\frac{\partial^{2} W_{m i d}^{U}(\bar{d})}{\partial \bar{d}^{2}}= & -\frac{2(\gamma+\omega)^{2}}{[k+(\gamma+\omega) \bar{d}]^{2}} \cdot \frac{k-(1+\eta) \bar{d}}{(1+\eta+\gamma+\omega) \bar{d}}-\frac{2(\gamma+\omega)}{k+(\gamma+\omega) \bar{d}} \cdot \frac{k}{(1+\eta+\gamma+\omega)\left(\bar{d}^{2}\right)} \\
& -\frac{\gamma+\omega}{(1+\eta)(1+\eta+\gamma+\omega)(\bar{d})^{2}} \cdot \frac{2[k-(1+\eta) \bar{d}]^{2}}{[k-(1+\eta) \bar{d}] \bar{d}}
\end{aligned}
$$

This expression shows that $\partial^{2} W_{m i d}^{U}(\bar{d}) / \partial \bar{d}^{2}<0$ holds, that is, $W_{m i d}^{U}(\bar{d})$ is strictly concave with respect to $\bar{d}$, if $\bar{d}<k /(1+\eta)$.

\section{Proof of statement (v)}

From Eq. (A.21), we have

$$
\lim _{\bar{d} \rightarrow \frac{k}{2+\eta}} \frac{\partial W_{l o w}^{U}(\bar{d})}{\partial \bar{d}} \gtreqless 0 \Leftrightarrow 2 \gtreqless \eta(\eta+\gamma+\omega) .
$$

Recall that the slopes of $W_{\text {low }}^{U}(\bar{d})$ and $W_{m i d}^{U}(\bar{d})$ at $\bar{d}=k /(2+\eta)$ are equivalent, as shown in Lemma A.1(ii). With the use of this property, we obtain

$$
\lim _{\bar{d} \rightarrow \frac{k}{2+\eta}} \frac{\partial W_{l o w}^{U}(\bar{d})}{\partial \bar{d}}=\left.\frac{\partial W_{m i d}^{U}(\bar{d})}{\partial \bar{d}}\right|_{\bar{d}=\frac{k}{2+\eta}} \gtreqless 0 \Leftrightarrow 2 \gtreqless \eta(\eta+\gamma+\omega) .
$$




\section{Proof of statement (vi)}

We substitute Eqs. (23) and (24) into Eq. (A.22) to obtain

$$
\begin{aligned}
\frac{\partial W_{m i d}^{U}(\bar{d})}{\partial \bar{d}}= & \frac{2(\gamma+\omega)}{k+(\gamma+\omega) \bar{d}} \cdot \frac{k-(1+\eta) \bar{d}}{(1+\eta+\gamma+\omega) \bar{d}} \\
& +\frac{k-2(1+\eta) \bar{d}}{\bar{d}} \cdot \frac{\gamma+\omega}{\bar{d}(1+\eta)(1+\eta+\gamma+\omega)}
\end{aligned}
$$

We evaluate the expression in Eq. (A.23) at $\bar{d}=k /(1+\eta)$ and obtain

$$
\left.\frac{\partial W_{m i d}^{U}(\bar{d})}{\partial \bar{d}}\right|_{\bar{d}=\frac{k}{1+\eta}}=\frac{-(1+\eta) k}{(1+\eta)\left(\frac{k}{1+\eta}\right)^{2}} \cdot \frac{\gamma+\omega}{(1+\eta)(1+\eta+\gamma+\omega)}<0 .
$$

We use the results in Lemma A.1 to illustrate the graph of the social welfare function, as depicted in Fig. A.3.

[Figure A.3 here.]

From the figure, and the results in Lemma A.1, we find the following properties: First, the social welfare function is continuous with respect to $\bar{d}$. Second, from the results in Lemma A.1 (iii) and (iv), the social welfare function is maximized within the range of $[0, k /(1+\eta))$. Finally, from the results in Lemma A.1 (i), (ii), and (iv), social welfare is maximized within the range $[0, k /(2+\eta))$ or $[k /(2+\eta), k /(1+\eta))$, depending on the slope of $W^{U}(\bar{d})$ at $\bar{d}=k /(2+\eta)$.

Let $\bar{d}^{*, c}$ denote the optimal $\bar{d}$ under the coordinated rule. From the result in Lemma A.1, $\bar{d}^{*, c}$ lies within the range $(0, k /(2+\eta))$ if $2<\eta(\eta+\gamma+\omega)$ and range $[k /(2+\eta), k /(1+\eta))$ if $2 \geq \eta(\eta+\gamma+\omega)$.

First, consider the case of $2<\eta(\eta+\gamma+\omega)$. From Eq. (A.21), the optimal coordinated rule, denoted by $\bar{d}^{*, c}$, satisfies

$$
\frac{\partial W_{l o w}^{U}\left(\bar{d}^{*, c}\right)}{d^{*, c}}=0
$$

Thus, $\bar{d}^{*, c}$ is given by

$$
\bar{d}^{*, c}=\frac{1}{1+\eta+\gamma+\omega} \cdot \frac{k}{\eta} .
$$


Second, consider the case of $2 \geq \eta(\eta+\gamma+\omega)$. From Eq. (A.23), $\bar{d}^{*, c}$ satisfies

$$
\frac{\partial W_{m i d}^{U}\left(\bar{d}^{*, c}\right)}{\partial \bar{d}}=0 \Leftrightarrow 2(1+\eta)(1+\eta+\gamma+\omega)\left(\bar{d}^{*, c}\right)^{2}-(\gamma+\omega) k \bar{d}^{*, c}-k^{2}=0 .
$$

Solving this for $\bar{d}^{*, c}$ leads to:

$$
\bar{d}^{*, c}=\frac{(\gamma+\omega) k+k \sqrt{(\gamma+\omega)^{2}+8(1+\eta)(1+\eta+\gamma+\omega)}}{4(1+\eta)(1+\eta+\gamma+\omega)} .
$$

\section{A.4 Proof of Proposition 4}

Case of $\beta<1 /(1+\eta+\gamma+\omega)$

First, suppose that $\beta$ satisfies $\beta<1 /(1+\eta+\gamma+\omega)$. In this case, the following condition holds at $\beta=0$ :

$$
d^{*, u c}(0)>d^{*, c}(0) \Leftrightarrow \eta(\eta+\gamma+\omega)>1
$$

The above inequality holds, that is, $d^{*, u c}(0)>d^{*, c}(0)$ holds, under Assumption 2.

The following condition also holds under Assumption 2.

$$
d^{*, u c}\left(\frac{1}{1+\eta+\gamma}\right)>d^{*, c}\left(\frac{1}{1+\eta+\gamma+\omega}\right) \Leftrightarrow \eta(\eta+\gamma+\omega)>2 .
$$

Given that $d^{*, u c}(\beta)$ is non-increasing in $\beta$, we have $d^{*, u c}(1 /(1+\eta+\gamma+\omega))>$ $d^{*, u c}(1 /(1+\eta+\gamma))$. Thus, we obtain

$$
d^{*, u c}\left(\frac{1}{1+\eta+\gamma+\omega}\right)>d^{*, c}\left(\frac{1}{1+\eta+\gamma+\omega}\right) .
$$

We suppose that there is a $\beta$ that satisfies $d^{*, u c}(\beta)=d^{*, c}(\beta)$ within the range of $\beta \in(0,1 /(1+\eta+\gamma+\omega))$ and show a contradiction. Eqs. (A.24) and (A.25) indicate that the solution, if it exists, is multiple-valued. Solving $d^{*, u c}(\beta)=d^{*, c}(\beta)$ for $\beta$ leads to:

$$
d^{*, u c}(\beta)=d^{*, c}(\beta) \Leftrightarrow \beta=\frac{(1+\eta)^{2}(\eta+\gamma+\omega)-(1+\eta+\gamma+\omega)^{2} \eta}{(1+\eta+\gamma+\omega)^{2} \eta-(1+\eta)(\eta+\gamma+\omega)(1+\eta+\gamma)}
$$

showing that the solution is unique; this is a contradiction. Thus, we can conclude 
that the following holds:

$$
d^{*, u c}(\beta)>d^{*, c}(\beta) \forall \beta \in\left[0, \frac{1}{1+\eta+\gamma+\omega}\right) .
$$

Case of $1 /(1+\eta+\gamma+\omega)<\beta<1 /(1+\eta+\gamma)$

Second, suppose that $\beta$ satisfies $1 /(1+\eta+\gamma+\omega)<\beta<1 /(1+\eta+\gamma)$. In this case, we have

$$
d^{*, u c}(\beta)>d^{*, c}(\beta) \Leftrightarrow \frac{\eta(\eta+\gamma+\omega)-1}{1+\eta+\gamma}>\beta .
$$

The above inequality holds under Assumption 2 and $\beta<1 /(1+\eta+\gamma)$. Thus, we obtain

$$
d^{*, u c}(\beta)>d^{*, c}(\beta) \forall \beta \in\left(\frac{1}{1+\eta+\gamma+\omega}, \frac{1}{1+\eta+\gamma}\right) .
$$

Case of $1 /(1+\eta+\gamma)<\beta<1 /(1+\eta)$

Third, suppose that $\beta$ satisfies $1 /(1+\eta+\gamma)<\beta<1 /(1+\eta) \cdot d^{*, c}(\beta)$ is given by $d^{*, c}(\beta)=(1 /(1+\eta+\gamma+\omega)) \cdot(k / \eta)$, and $d^{*, u c}(\beta)=0$. Thus, we obtain

$$
d^{*, c}(\beta)=\frac{1}{1+\eta+\gamma+\omega} \cdot \frac{k}{\eta}>0=d^{*, u c}(\beta) \forall \beta \in\left(\frac{1}{1+\eta+\gamma}, \frac{1}{1+\eta}\right) .
$$

Case of $1 /(1+\eta)<\beta \leq 1$

Finally, suppose that $\beta$ satisfies $1 /(1+\eta)<\beta<1$. In this case, we have $d^{*, u c}(\beta)=$ $d^{*, c}(\beta)=0 \forall \beta \in(1 /(1+\eta), 1]$.

\section{A.5 Proof of Lemma 1}

Case of $\beta<1 /(1+\eta+\gamma+\omega)$

First, suppose that $\beta<1 /(1+\eta+\gamma+\omega)$ holds. For a given $d^{*, c}(\beta)$, there is an equilibrium characterized in Proposition 1(i). With the use of the optimal coordinated rule in Eq. (25) and the result in Proposition A.1, we can compute 
the social welfare as follows:

$$
\begin{aligned}
W^{*, c}(\beta) & =V_{(i)}^{\omega>0}\left(\bar{d}^{*, c}, \beta\right) \\
& =2 \ln \left[\frac{(1+\eta)(\eta+\gamma+\omega)}{1+\eta+\gamma+\omega} \cdot \frac{k}{\eta}\right]+\ln \left[\frac{\beta}{(1+\beta)^{2}(1+\eta+\gamma+\omega)}\right],
\end{aligned}
$$

where the subscript (i) corresponds to the statements in Proposition 1; the superscript $\omega>0$ indicates the coordinated debt rule case.

\section{Case of $1 /(1+\eta+\gamma+\omega)<\beta<1 /(1+\eta)$}

Second, suppose that $1 /(1+\eta+\gamma+\omega)<\beta<1 /(1+\eta)$ holds. For a given $d^{*, c}(\beta)$, there is an equilibrium characterized in Proposition 1(iiib). With the use of the optimal coordinated rule in Eq. (25) and the result in Proposition A.4, we can compute the social welfare as follows:

$$
W^{*, c}(\beta)=V_{(i i i, b)}^{\omega>0}\left(\bar{d}^{*, c}\right)=2 \ln \left[\frac{\eta(\eta+\gamma+\omega)}{2(1+\eta+\gamma+\omega)} \cdot \frac{k}{\eta}\right]
$$

Case of $1 /(1+\eta)<\beta \leq 1$

Third, suppose that $1 /(1+\eta) \leq \beta<1$ holds. For a given $d^{*, c}(\beta)$, there is an equilibrium characterized in Proposition A.7(vi). With the use of the optimal coordinated rule in Eq. (25) and the result in Proposition A.7, we can compute the social welfare as follows:

$$
W^{*, c}(\beta)=V_{(v i)}^{\omega>0}=2 \ln \left(\frac{k}{2}\right) .
$$

Case of $\beta=1 /(1+\eta+\gamma+\omega)$

Fourth, suppose that $\beta=1 /(1+\eta+\gamma+\omega)$ holds. For a given $d^{*, c}(\beta)$, there are equilibria characterized in Proposition 1 (i), (ii), and (iiib). With the use of the optimal coordinated rule in Eq. (25), the result in Proposition A.2 and Eqs. (A.27) and (A.28), we have:

$$
\begin{aligned}
& \inf _{d} V_{(i i)}^{\omega>0}\left(\bar{d}^{*, c}, d\right)=V_{(i)}^{\omega>0}\left(\bar{d}^{*, c}, \beta=\frac{1}{1+\eta+\gamma+\omega}\right)=2 \ln \left[\frac{(1+\eta)(\eta+\gamma+\omega)}{(2+\eta+\gamma+\omega)(1+\eta+\gamma+\omega)} \cdot \frac{k}{\eta}\right], \\
& \sup _{d} V_{(i i)}\left(\bar{d}^{*, c}, d\right)=V_{(i i i, b)}\left(\bar{d}^{*, c}\right)=2 \ln \left[\frac{\eta(\eta+\gamma+\omega)}{2(1+\eta+\gamma+\omega)} \cdot \frac{k}{\eta}\right] .
\end{aligned}
$$


Therefore, the social welfare in the present case becomes:

$$
W^{*, c}(\beta) \in\left[V_{(i)}^{\omega>0}\left(\bar{d}^{*, c}, \beta=\frac{1}{1+\eta+\gamma+\omega}\right), V_{(i i i, b)}^{\omega>0}\left(\bar{d}^{*, c}\right)\right]
$$

Case of $\beta=1 /(1+\eta)$

Finally, suppose that $\beta=1 /(1+\eta)$ holds. For a given $d^{*, c}(\beta)$, there are equilibria characterized in Proposition 1(iii,a), (iv), and (vi). Note that $d^{*, c}(\beta)<k /(2+\eta)$ holds. With the use of the optimal coordinated rule in Eq. (25), the result in Proposition A.6 and Eqs. (25) and (A.27), we have:

$$
\begin{aligned}
& \inf _{d} V_{(v)}^{\omega>0}\left(\bar{d}^{*, c}, d\right)=V_{(i i i, b)}^{\omega>0}\left(\bar{d}^{*, c}\right)=2 \ln \left[\frac{\eta(\eta+\gamma+\omega)}{2(1+\eta+\gamma+\omega)} \cdot \frac{k}{\eta}\right], \\
& \sup _{d} V_{(v)}\left(\bar{d}^{*, c}, d\right)=V_{(v i)}^{\omega>0}=2 \ln \left(\frac{k}{2}\right) .
\end{aligned}
$$

Therefore, the social welfare in the present case becomes:

$$
W^{*, c}(\beta) \in\left[V_{(i i i, b)}^{\omega>0}\left(\bar{d}^{*, c}\right), V_{(v i)}^{\omega>0}\right]
$$

\section{A.6 Proof of Proposition 5}

Case of $\beta>1 /(1+\eta+\gamma+\omega)$

First, suppose that $1 /(1+\eta)<\beta \leq 1$ holds. Based on the results established in Proposition 2 and Lemma 1, we compare the social welfare between the two cases (that is, the coordinated and uncoordinated cases) and obtain

$$
W^{*, c}(\beta)=V_{(v i)}^{\omega>0}=2 \ln \left(\frac{k}{2}\right)=W^{*, u c}(\beta)
$$

Second, suppose that $1 /(1+\eta+\gamma)<\beta<1 /(1+\eta)$ holds. Following the method used in the previous case, we obtain

$$
W^{*, c}(\beta)=V_{(i i i, b)}^{\omega>0}\left(\bar{d}^{*, c}\right)=2 \ln \left[\frac{\eta(\eta+\gamma+\omega)}{2(1+\eta+\gamma+\omega)} \cdot \frac{k}{\eta}\right]<2 \ln \left(\frac{k}{2}\right)=W^{*, u c}(\beta) .
$$

Third, suppose that $1 /(1+\eta+\gamma+\omega)<\beta<1 /(1+\eta+\gamma)$ holds. Notice that we have $W^{*, c}(\beta)=V_{(i i i, b)}^{\omega>0}$ and $W^{*, u c}(\beta)=V_{(i i i, a)}^{\omega=0}\left(\bar{d}_{L}(\beta)\right)$, and note that 
$V_{(i i i, a)}^{\omega=0}\left(\bar{d}_{L}(\beta)\right)$ is increasing in $\beta$. Then we have

$$
\left.V_{(i i i, a)}^{\omega=0}\left(\bar{d}_{L}(\beta)\right)\right|_{\beta<1 /(1+\eta+\gamma)}<V_{(i i i, a)}^{\omega=0}\left(\bar{d}_{L}\left(\frac{1}{1+\eta+\gamma}\right)\right)=2 \ln \left(\frac{k}{2+\eta}\right)
$$

where we have

$$
2 \ln \left(\frac{k}{2+\eta}\right)<V_{(i i i, b)}^{\omega>0}\left(\bar{d}^{*, c}\right) \Leftrightarrow 2<\eta(\eta+\gamma+\omega)
$$

This condition holds under Assumption 2. Therefore, we conclude that $W^{*, c}(\beta)>$ $W^{*, u c}(\beta)$ holds.

Case of $\beta<1 /(1+\eta+\gamma+\omega)$

Finally, suppose that $\beta<1 /(1+\eta+\gamma+\omega)$ holds. The results established in Proposition 2 and Lemma 1 lead to $W^{*, c}(\beta)=V_{(i)}^{\omega>0}\left(\bar{d}^{*, c}\right)$, and $W^{*, u c}(\beta)=$ $V_{(i i i, a)}^{\omega=0}\left(\bar{d}_{L}(\beta)\right)$, We compare $W^{*, c}(\beta)$ and $W^{*, u c}(\beta)$ and obtain

$$
W^{*, c}(\beta) \gtreqless W^{*, u c}(\beta) \Leftrightarrow-\phi_{L} \beta \gtreqless \phi_{H},
$$

where $\phi_{L}$ and $\phi_{H}\left(>\phi_{L}\right)$ are defined by

$$
\begin{aligned}
\phi_{L} & \equiv(1+\eta+\gamma+\omega) \eta \sqrt{(1+\eta+\gamma)(1+\eta+\gamma+\omega)}-(1+\eta)(1+\eta+\gamma)(\eta+\gamma+\omega) \\
\phi_{H} & \equiv(1+\eta+\gamma+\omega) \eta \sqrt{(1+\eta+\gamma)(1+\eta+\gamma+\omega)}-(1+\eta)^{2}(\eta+\gamma+\omega)>\phi_{L} .
\end{aligned}
$$

Focusing on the level of $\phi_{L}$, we divide the current case into four parts. First, when $\phi_{L}>0$ holds, we can reformulate Eq. (A.29) as $W^{*, c}(\beta) \gtreqless W^{*, u c}(\beta) \Leftrightarrow$ $\beta \lesseqgtr-\phi_{H} / \phi_{L}$. We have $-\phi_{H} / \phi_{L}<0$ because $\phi_{H}>\phi_{L}>0$ holds. Therefore, we conclude that $W^{*, c}(\beta)<W^{*, u c}(\beta)$ holds $\forall \beta<1 /(1+\eta+\gamma+\omega)$.

Second, when $\phi_{L}=0$ holds, we can rewrite Eq. (A.29) as $W^{*, c}(\beta) \gtreqless W^{*, u c}(\beta) \Leftrightarrow$ $0 \gtreqless \phi_{H}$. Given that $\phi_{H}>\phi_{L}=0$ holds, we immediately find that $W^{*, c}(\beta)<$ $W^{*, u c}(\beta)$ holds $\forall \beta<1 /(1+\eta+\gamma+\omega)$.

Third, when $\phi_{H}>0>\phi_{L}$ holds, we have $-\phi_{H} / \phi_{L}>0$. Given this condition, we can reformulate Eq. (A.29) as $W^{*, c}(\beta) \gtreqless W^{*, u c}(\beta) \Leftrightarrow \beta \gtreqless-\phi_{H} / \phi_{L}$. Thus, we obtain

$$
W^{*, c}(\beta)\left\{\begin{array}{l}
<W^{*, u c}(\beta) \text { if } \frac{1}{1+\eta+\gamma+\omega} \leq-\frac{\phi_{H}}{\phi_{L}} \\
>W^{*, u c}(\beta) \text { if }-\frac{\phi_{H}}{\phi_{L}}<\beta<\frac{1}{1+\eta+\gamma+\omega} \\
\leq W^{*, u c}(\beta) \text { if } \beta \leq-\frac{\phi_{H}}{\phi_{L}}<\frac{1}{1+\eta+\gamma+\omega}
\end{array}\right.
$$


Finally, when $0 \geq \phi_{H}$ holds, we have $0 \geq \phi_{H}>\phi_{L}$. Given this condition, we can write Eq. (A.29) as $W^{*, c}(\beta) \gtreqless W^{*, u c}(\beta) \Leftrightarrow \beta \gtreqless-\phi_{H} / \phi_{L}$. It should be noted that $-\phi_{H} / \phi_{L} \leq 0$ holds because $0 \geq \phi_{H}>\phi_{L}$. Therefore, we conclude that $W^{*, c}(\beta) \geq W^{*, u c}(\beta)$ holds $\forall \beta<1 /(1+\eta+\gamma+\omega)$.

\section{References}

[1] Arawatari, R., and Ono, T., 2017. Inequality and public debt: A positive analysis. Review of International Economics 25, 1155-1173.

[2] Arawatari, R., and Ono, T., 2020a. Public Debt Rule Breaking by Timeinconsistent Voters. Working Paper Series No.55, Faculty of Economics, Doshisha University.

[3] Arawatari, R., and Ono, T., 2020b. Age gap in voter turnout and size of government debt. International Tax and Public Finance 27, 435-460.

[4] Arcalean, C., 2017. International tax competition and deficit bias. Economic Inquiry 55, 51-72.

[5] Arcalean, C., 2018. Dynamic fiscal competition: Political economy theory. Journal of Public Economics 164, 211-224.

[6] Banerji, A., Narkbu, B., John, J., Kinda, T., Saksonovs, S., Schoelermann, H., Wu, T., 2015. Building a better union: incentivizing structural reforms in the EA. IMF Working Paper, WP/15/201. International Monetary Fund.

[7] Beqiraj, E., Fedeli, S., Forte, F., 2018. Public debt sustainability: An empirical study on OECD countries. Journal of Macroeconomics 58, 238-248.

[8] Bhanot, K., Burns, N., Hunter, D., Williams, M., 2014. News spillovers from the Greek debt crisis: Impact on the Eurozone financial sector. Journal of Banking and Finance 38, 51-63.

[9] Bisin, A., Lizzeri, A., and Yariv, L., 2015. Government policy with time inconsistent voters. American Economic Review 105, 1711-1737.

[10] Chang, R., 1990. International coordination of fiscal deficits. Journal of Monetary Economics 25, 347-366.

[11] Coate, S., and Milton, R.T., 2019. Optimal fiscal limits with overrides. Journal of Public Economics 174, 76-92.

[12] Collignon, S., 2012. Fiscal policy rules and the sustainability of public debt in Europe. International Economic Review 53, 539-567.

[13] Dovis, A., and Kirpalani, R., 2020. Fiscal rules, bailouts, and reputation in federal governments. American Economic Review 110, 860-888. 
[14] EEAG, 2011. New crisis mechanism for the Euro Area. The EEAG Report on the European Economy, CESifo, Munich, 2011, pp. 71-96.

[15] European Commission (2019, July 3). The Commission concludes that an excessive deficit procedure is no longer warranted for Italy at this stage (Press release).

[16] Eyraud, L., Debrun, X., Hodge, A., Lledo, V., Pattillo, C., 2018. Secondgeneration fiscal rules: Balancing simplicity, flexibility, and enforceability. IMF Staff Discussion Note: SDN/18/04.

[17] Halac, M., Yared, P., 2018. Fiscal rules and discretion in the world economy. American Economic Review 108, 2305-2334.

[18] Halac, M., and Yared, P., 2020. Fiscal rules and discretion under limited enforcement. NBER Working Paper No. 25463

[19] Higgins, M., and Klitgaard, T., 2011. Saving imbalance and the Euro area sovereign debt crisis. Current Issues in Economics and Finance 17, 1-11, Federal Reserve Bank of New York.

[20] Janeba, E., and Todtenhauput, M., 2018. Fiscal competition and public debt Journal of Public Economics 168, 47-61.

[21] Laibson, D., 1997. Golden eggs and hyperbolic discounting. Quarterly Journal of Economics 112, 443-478.

[22] Lledo, V., Yoon, S., Fang, X., Mbaye, S., Kim, Y., 2017. Fiscal rules at a glance. International Monetary Fund. https://pdfs.semanticscholar.org/97b1/91565d9c9e723df01986a483d130aae5b35a.pdf (Accessed on August 23, 2020).

[23] Persson, T., and Svensson, L.E.O., 1989. Why a stubborn conservative would run a deficit: Policy with time-inconsistent preferences. Quarterly Journal of Economics 104, 325-345.

[24] Phelps, E.S., Pollak, R.A., 1968. On second-best national saving and gameequilibrium growth. Review of Economic Studies 35, 185-199.

[25] Song, Z., Storesletten, K., Zilibotti, F., 2012. Rotten parents and disciplined children: A politico-economic theory of public expenditure and debt. Econometrica 80, 2785-2803.

[26] Strotz, R.H., 1956. Myopia and inconsistency in dynamic utility maximization. Review of Economic Studies 23, 165-180.

[27] Wyplosz, C., 2013. Fiscal rules: Theoretical issues and historical experiences. In: Alesina, A., Giavazzi, F. (Eds), Fiscal policy after the financial crisis. University of Chicago Press, 495-525.

[28] Yared, P., 2019. Rising government debt: Causes and solutions for a decadesold trend. Journal of Economic Perspectives 33, 115-140. 


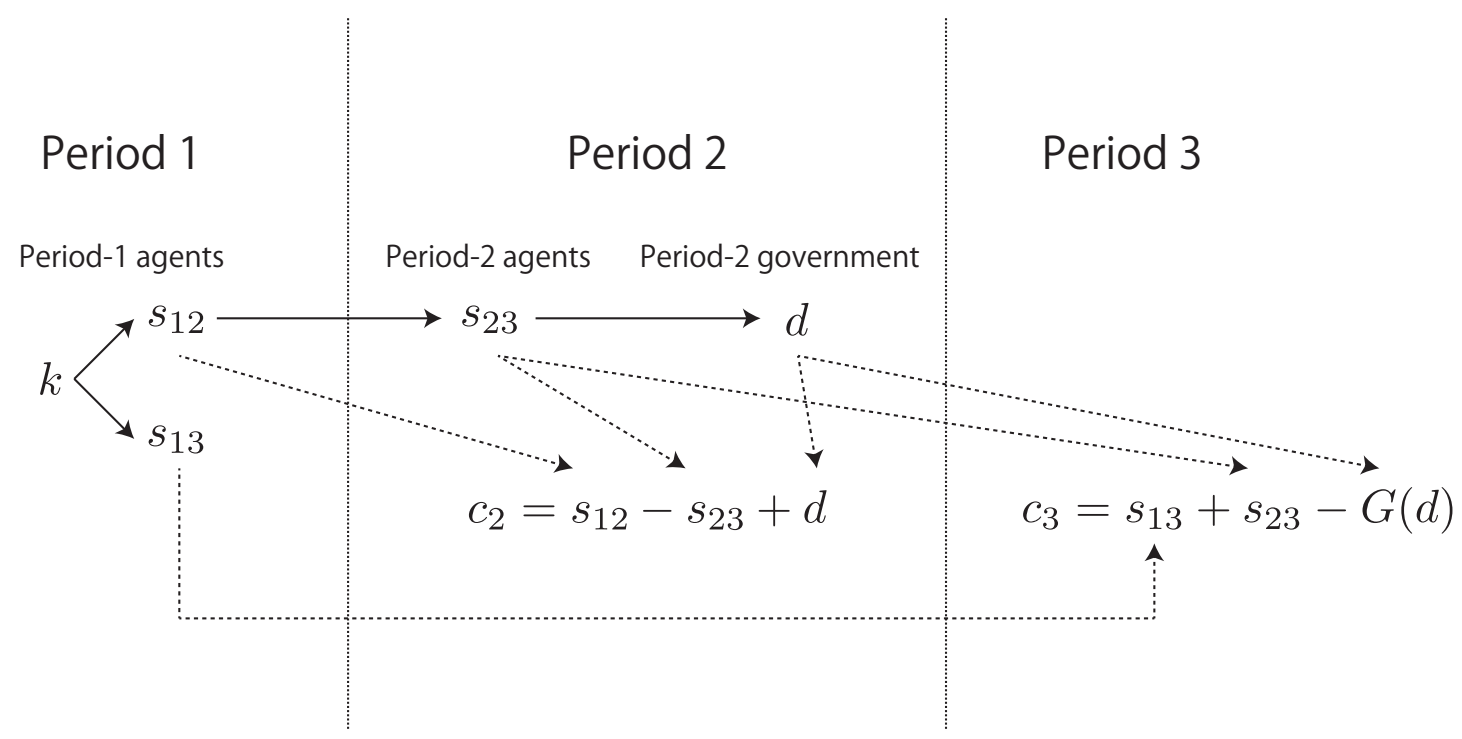

Figure 1: Timing of events. 
(a)

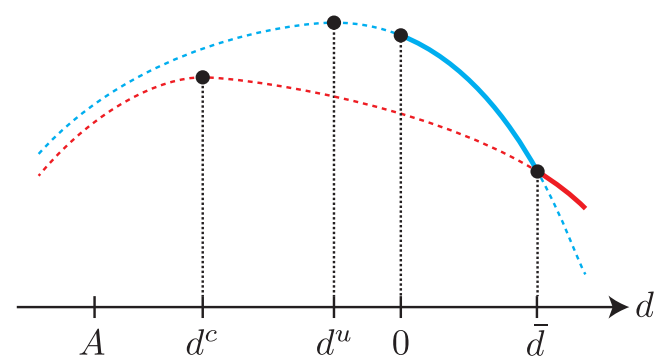

(c)

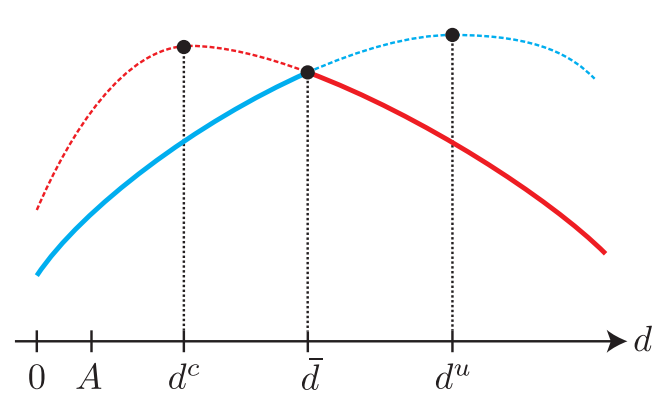

(b)

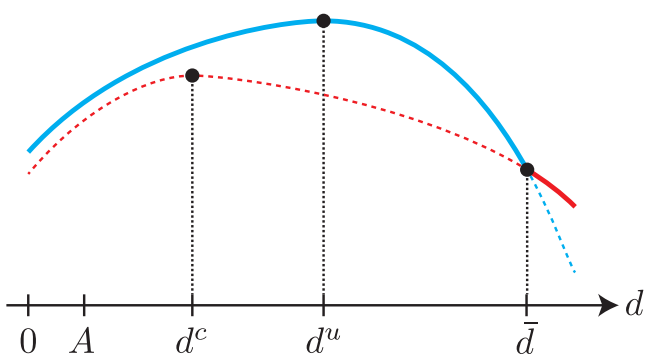

(d)

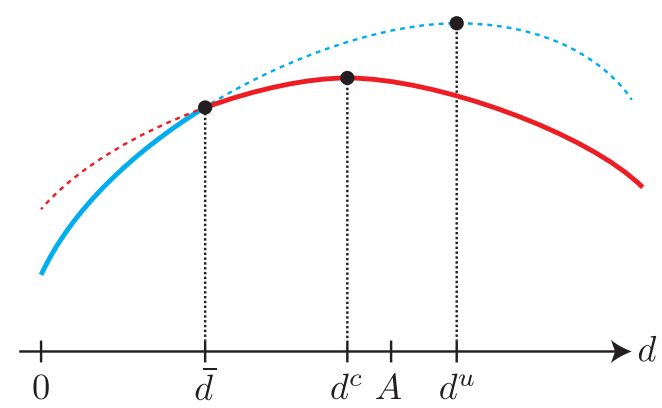

Figure 2: Illustration of the period-2 government's objective function when $d^{u}\left(s_{12}, s_{23}\right) \leq 0 \leq \bar{d}$ (panel (a)), $0<d^{u}\left(s_{12}, s_{23}\right)<\bar{d}$ (panel (b)), $A\left(s_{12}, s_{23}\right) \leq \bar{d} \leq$ $d^{u}\left(s_{12}, s_{23}\right)$ (panel (c)), and $\bar{d}<A\left(s_{12}, s_{23}\right)$ (panel (d)). 


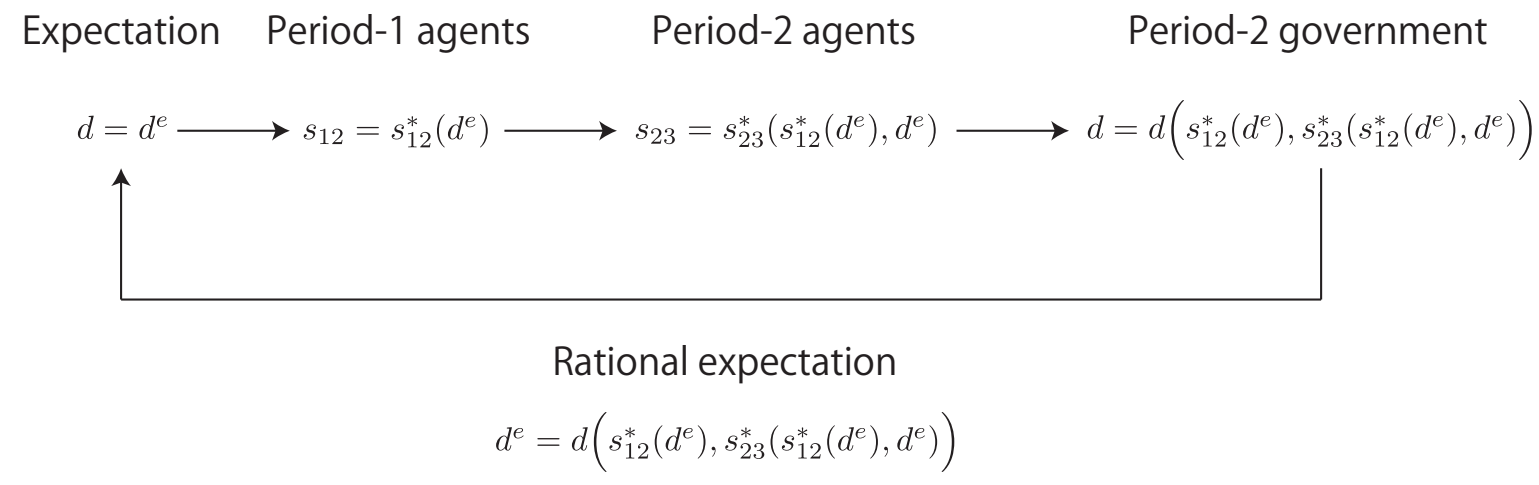

Figure 3: Rational expecations equilibrium. 


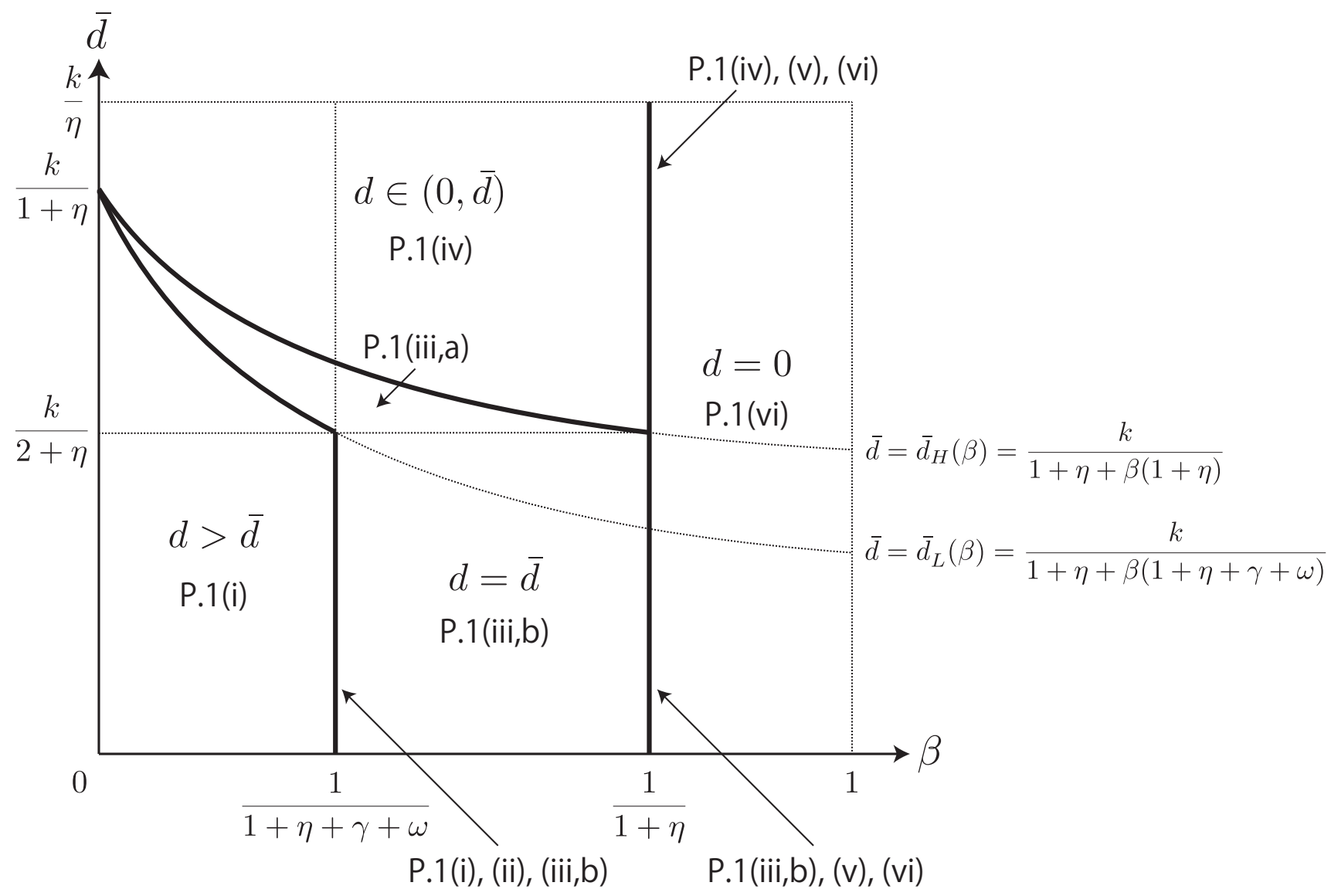

Figure 4: Classification of the equilibrium states according to the level of public debt for a given debt rule. The horizontal axis takes $\beta$; the vertical axis takes $\bar{d}$. 

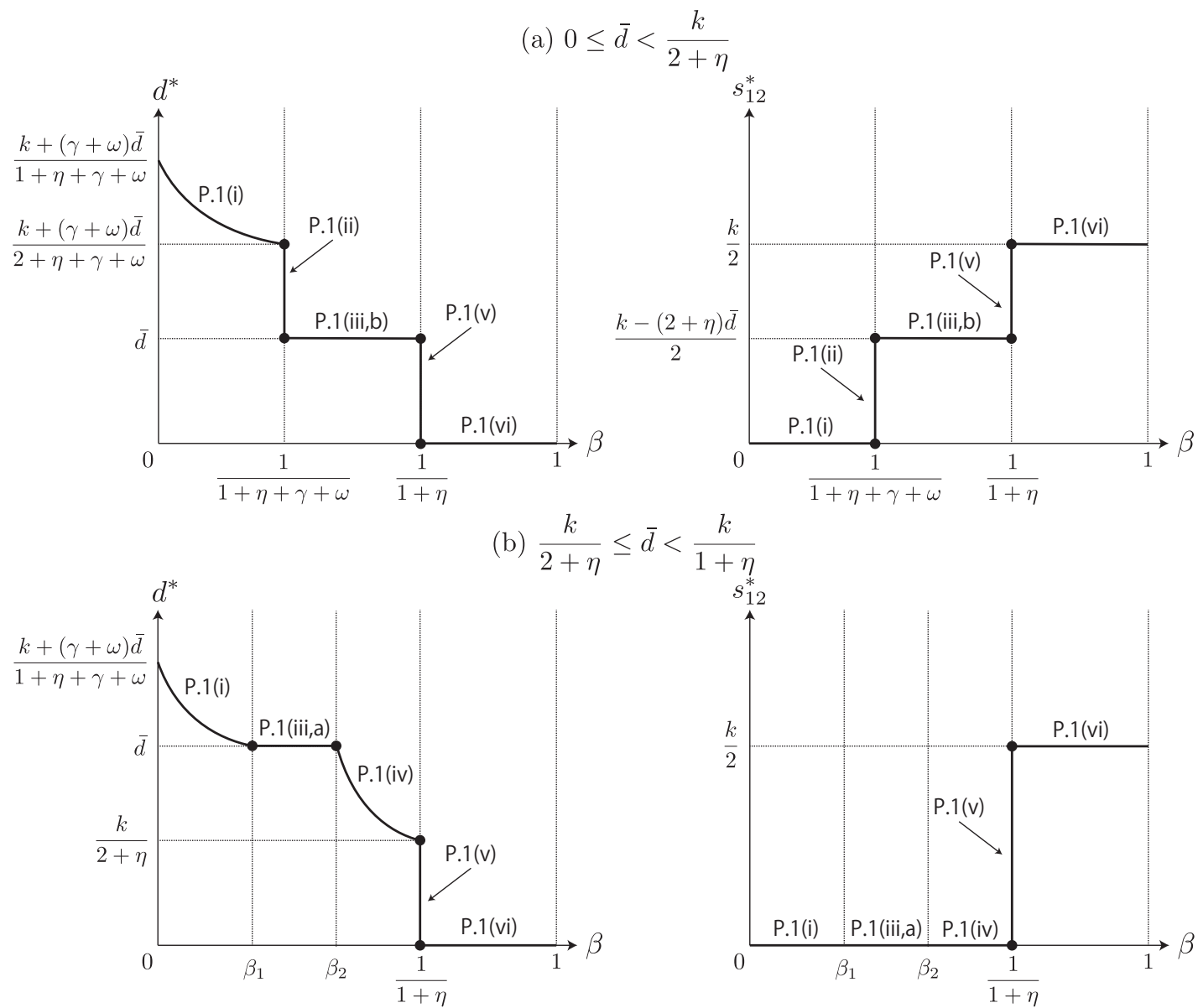

(b) $\frac{k}{2+\eta} \leq \bar{d}<\frac{k}{1+\eta}$

(c) $\frac{k}{1+\eta} \leq \bar{d}$
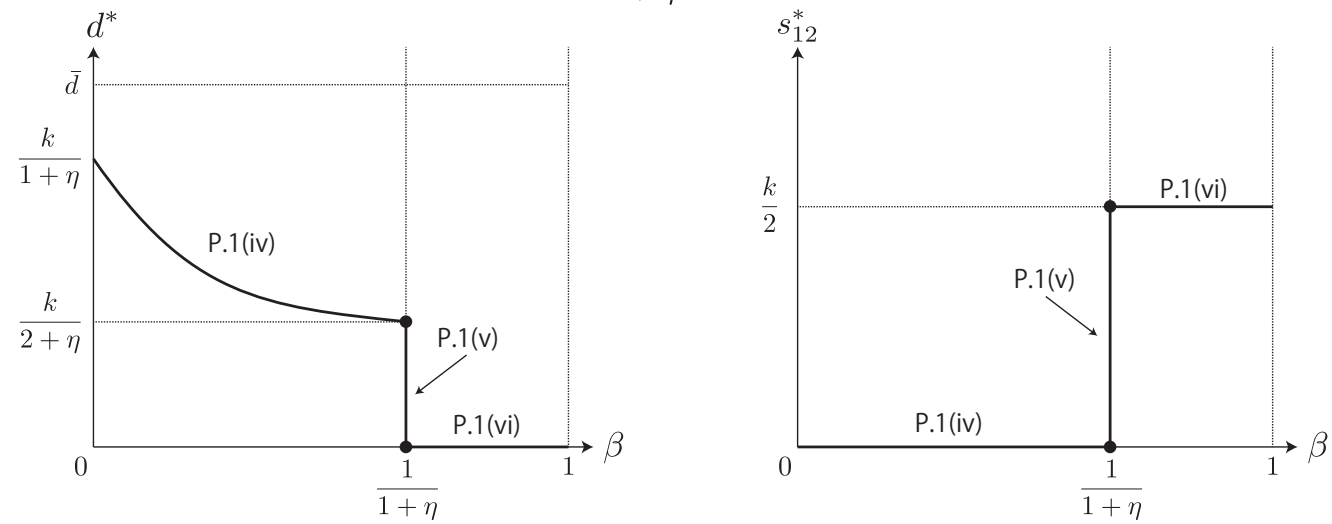

Figure 5: The equilibrium debt according to $\beta$. $\beta_{1} \equiv \frac{1}{1+\eta+\gamma} \cdot \frac{k-(1+\eta) \bar{d}}{\bar{d}}, \beta_{2} \equiv$ $\frac{1}{1+\eta} \cdot \frac{k-(1+\eta) \bar{d}}{\bar{d}}$. 


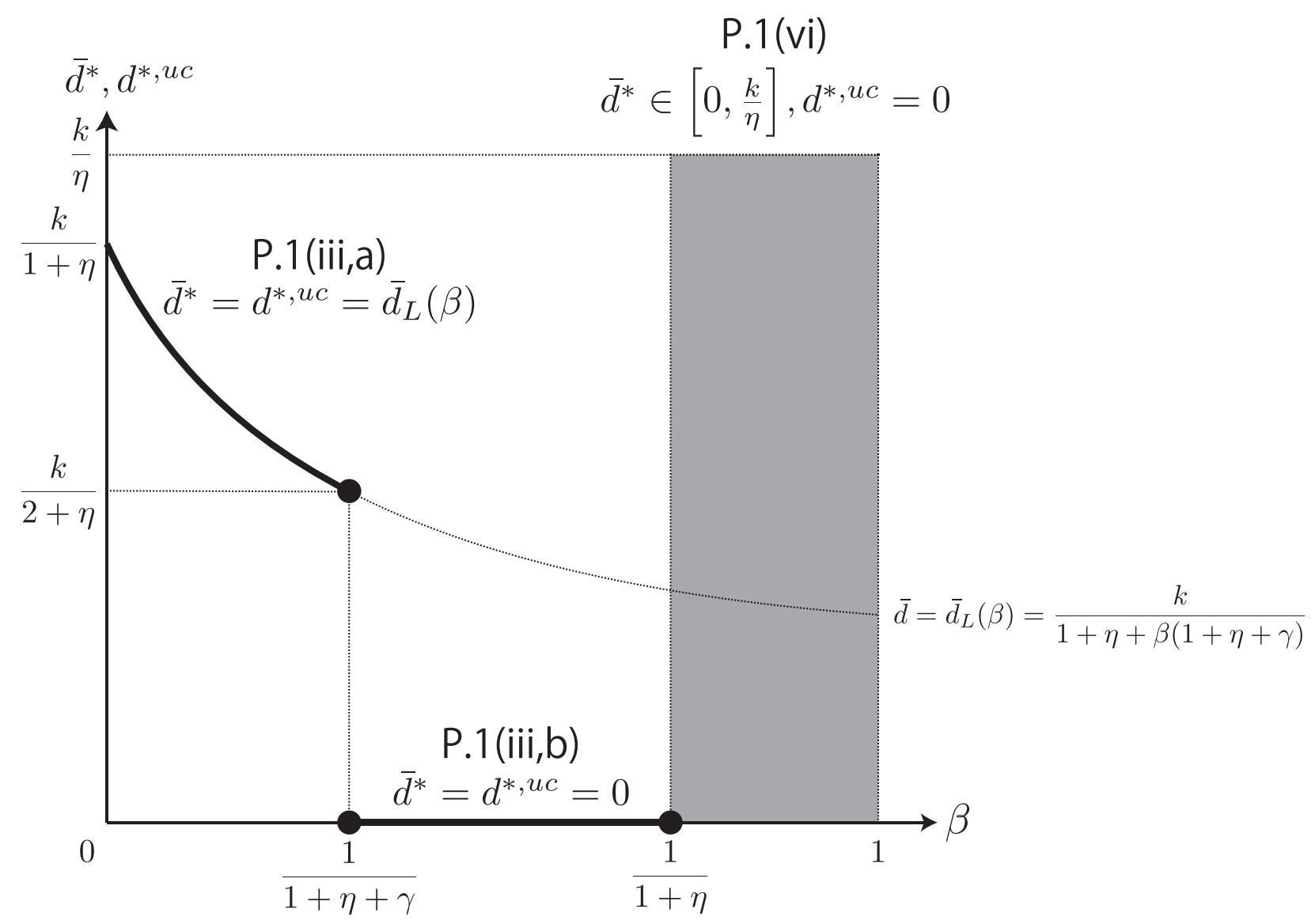

Figure 6: The optimal uncoordinated debt ceiling for the period-1 selves according to $\beta$. 


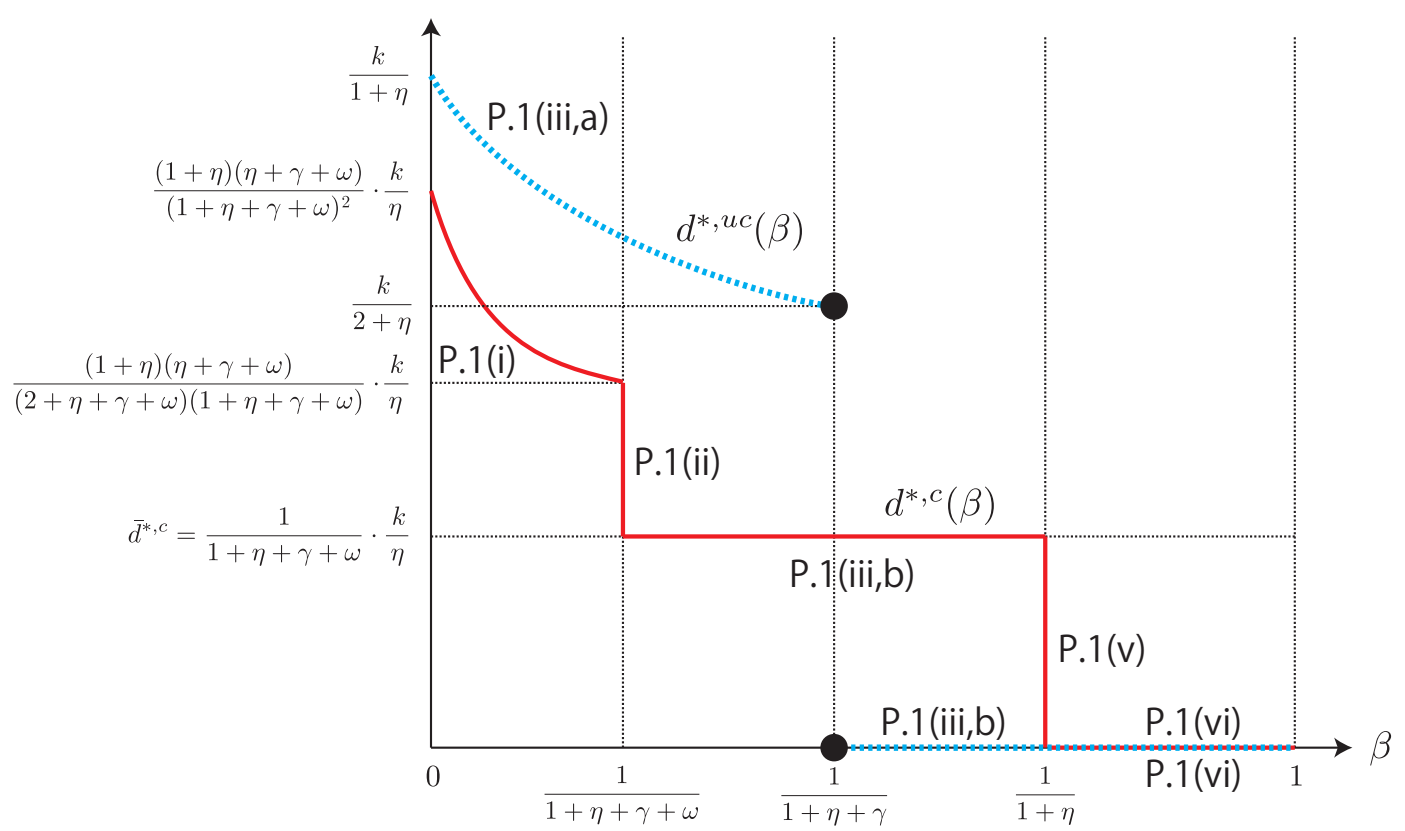

Figure 7: The debt levels in the uncoordinated case (plotted by the blue dotted curve) and in the coordinated case (plotted by the red solid curve) 


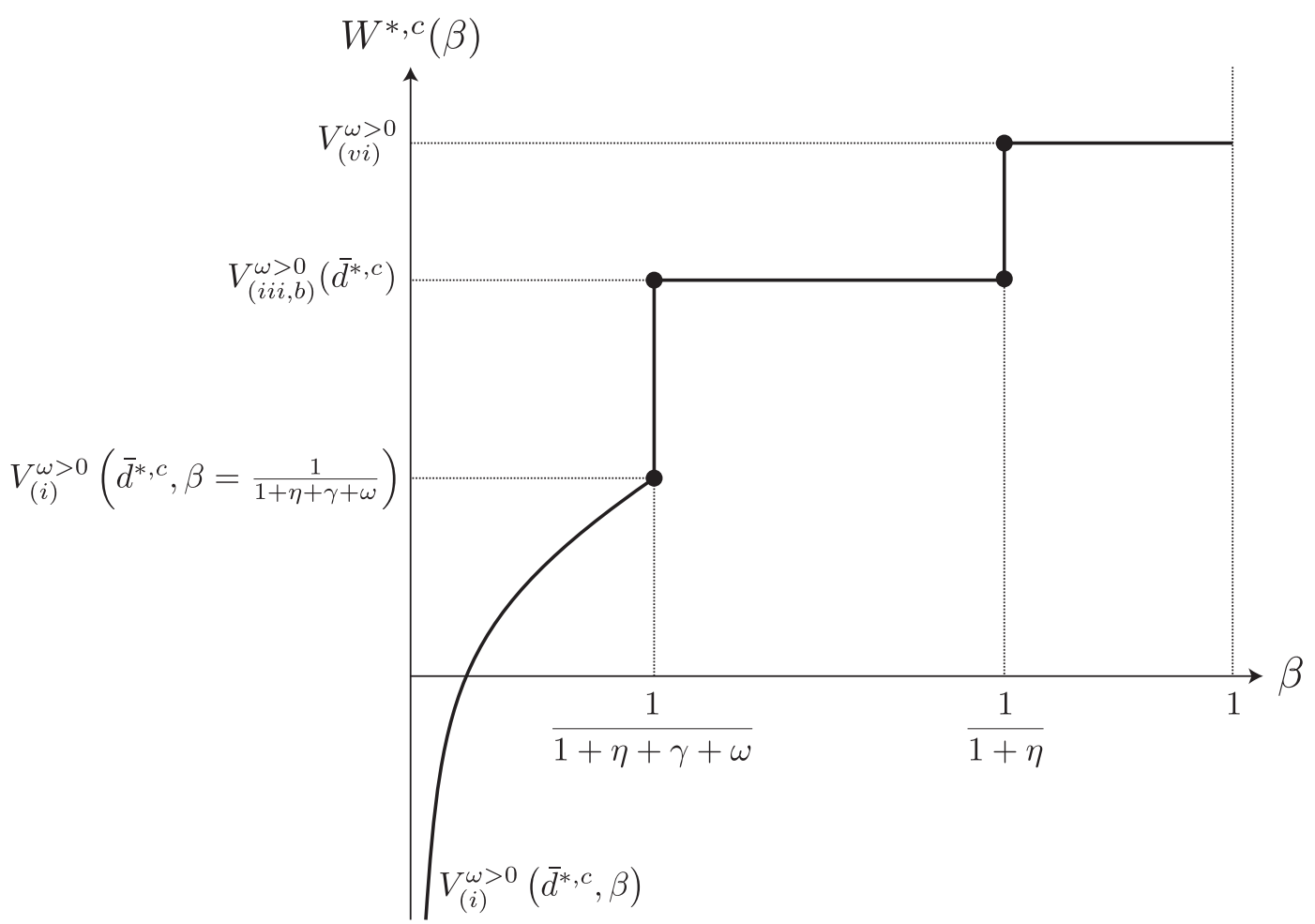

Figure 8: Social welfare in the coordinated case. 

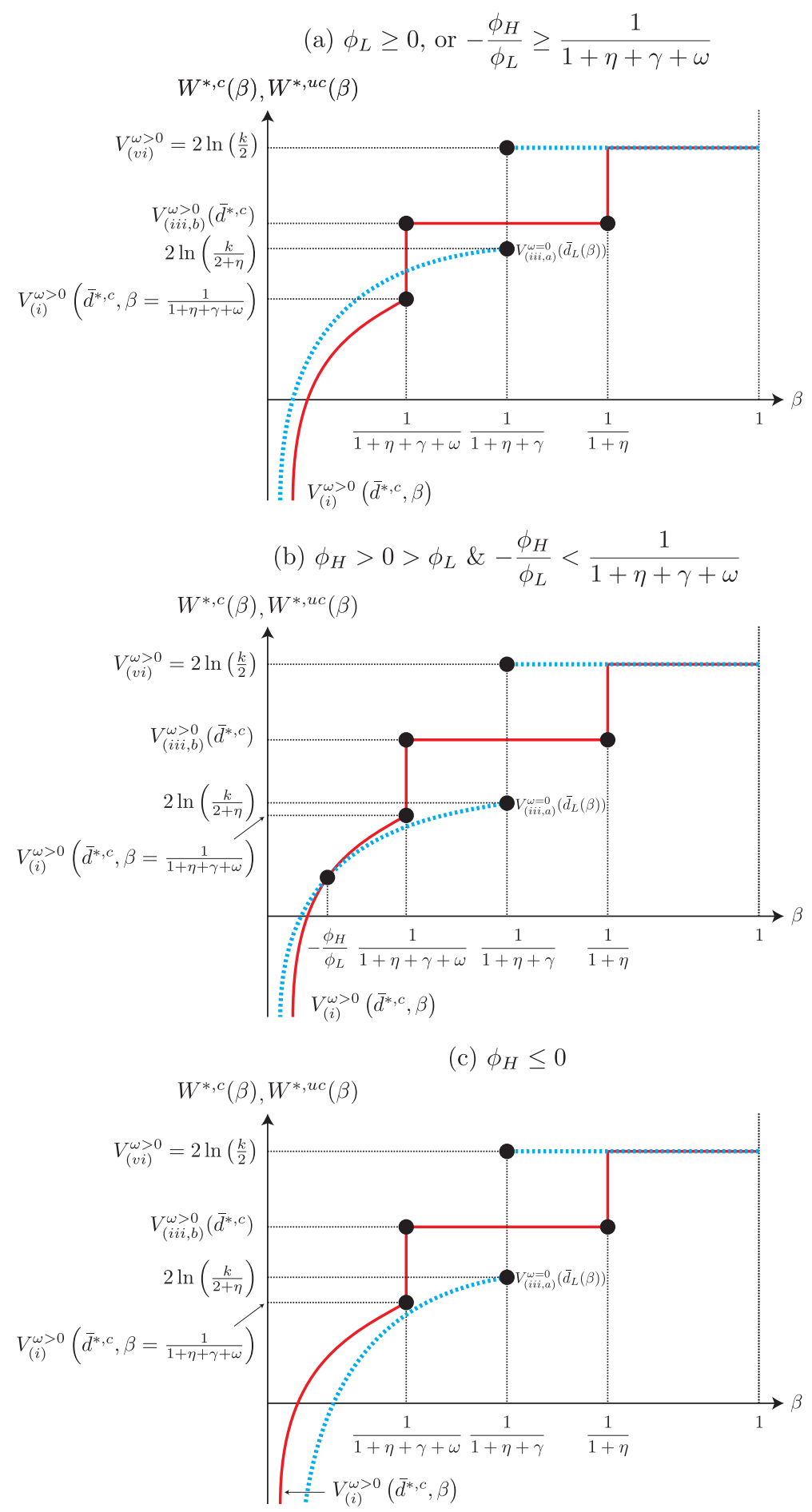

Figure 9: Social welfare in the uncoordinated case (plotted by the blue curve) and in the coordinated case (plotted by the red solid curve). 
(a) $\eta=0.5$

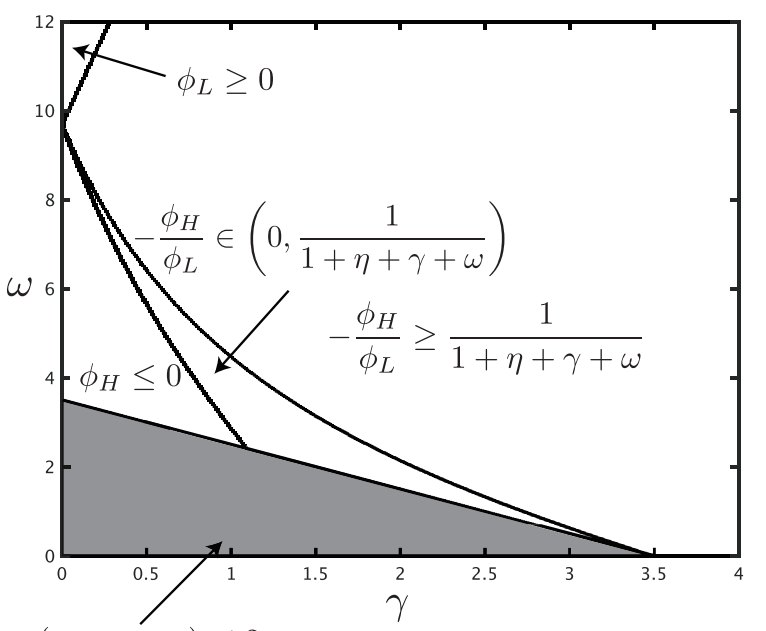

$\eta(\eta+\gamma+\omega) \leq 2$ (b) $\eta=1.0$

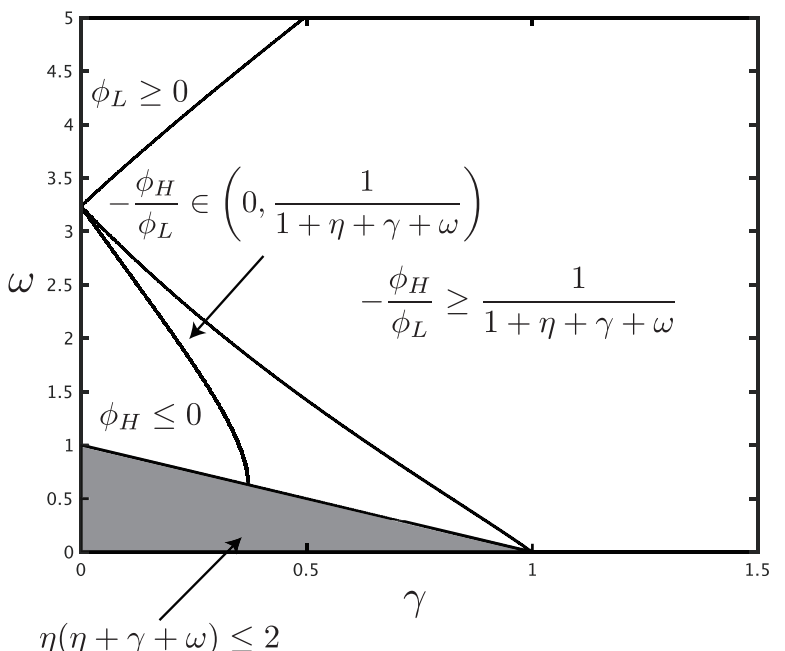

(c) $\eta=1.5$

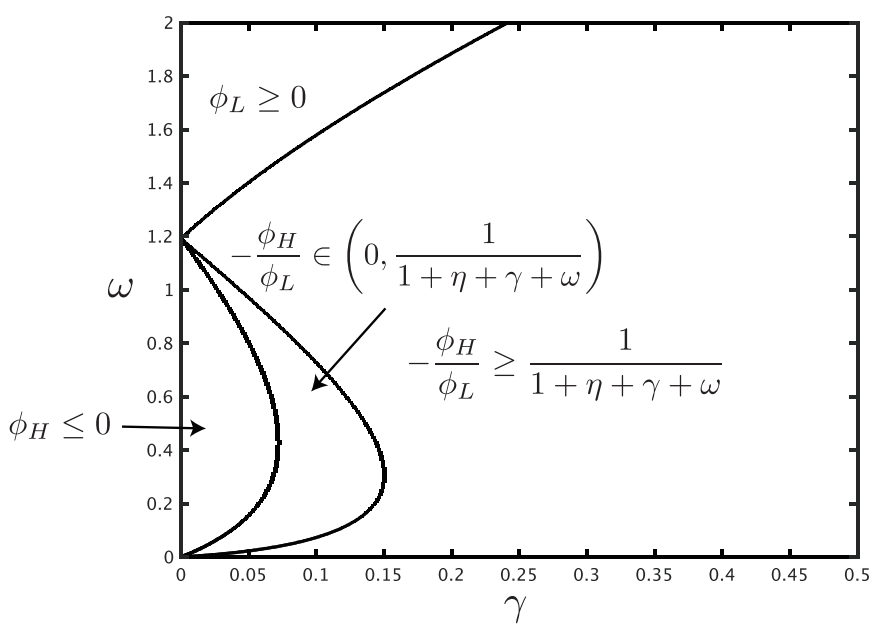

Figure 10: A set of $(\gamma, \omega)$ that satisfies the conditions in Proposition 5(ii) for three cases: $\eta=0.5$ (panel (a)), $\eta=1.0($ panel (b)), and $\eta=1.5($ panel (c)) 
(a)

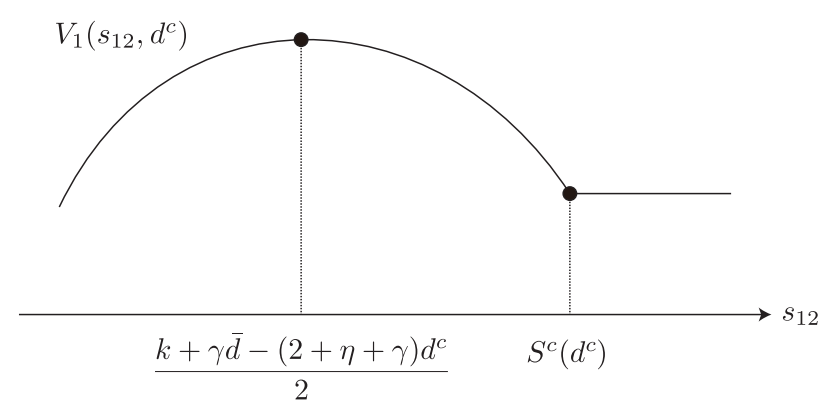

(b)

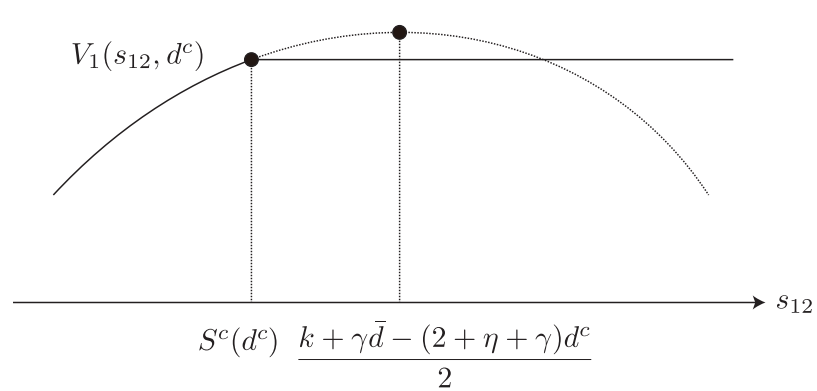

Figure A.1: Illustration of the period-1 selves' utility, $V_{1}\left(s_{12}, d^{c}\right)$, when $\left[k+\gamma \bar{d}-(2+\eta+\gamma) d^{c}\right] / 2 \leq S^{c}\left(d^{c}\right) \quad$ (panel (a)) and $\left[k+\gamma \bar{d}-(2+\eta+\gamma) d^{c}\right] / 2>S^{c}\left(d^{c}\right)$ (panel (b)). 
(a)

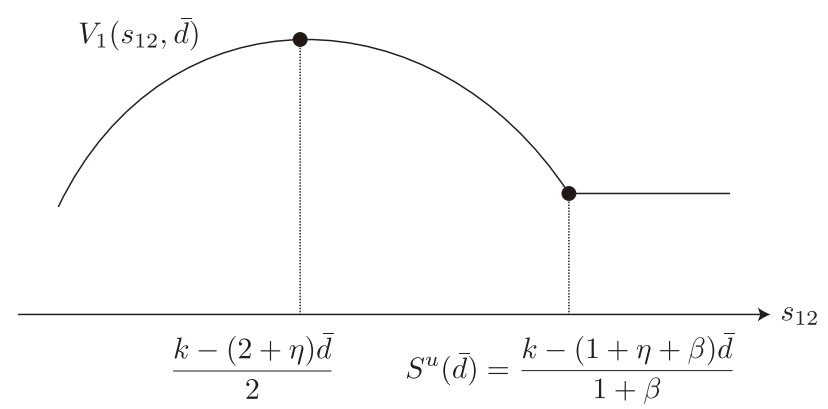

(b)

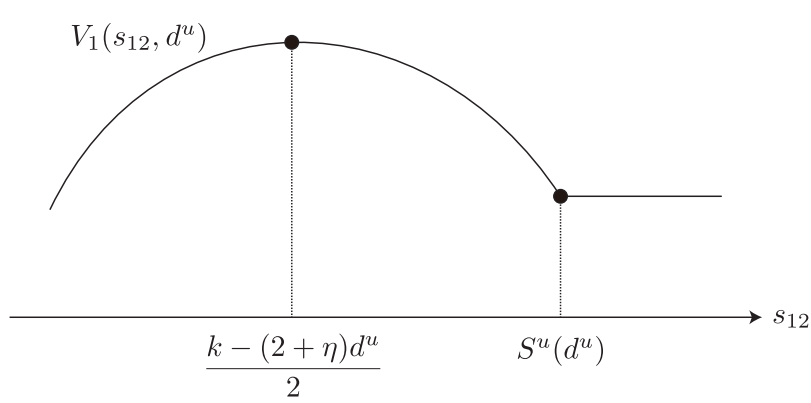

(c)

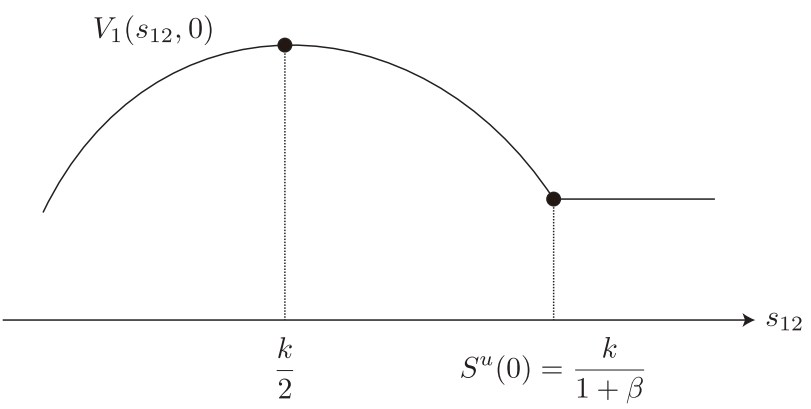

Figure A.2: Illustration of the period-1 selves' utility when $d^{e}=\bar{d}$ (panel (a)), $d^{e}=d^{u}($ panel $(\mathrm{b}))$, and $d^{e}=0($ panel $(\mathrm{c}))$ 
(a)

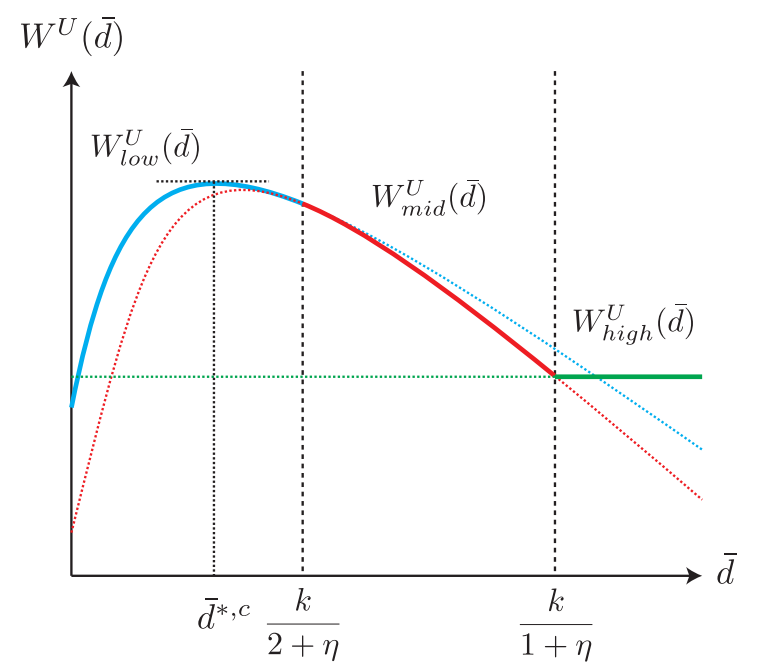

(b)

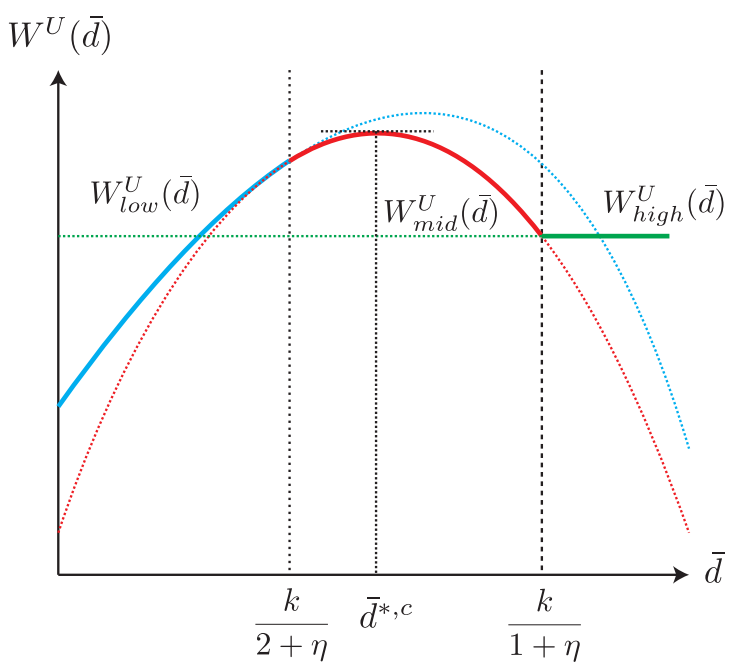

Figure A.3: Illustration of the social welfare function in the coordinated case: case of $2<\eta(\eta+\gamma+\omega)$ in panel (a) and case of $2 \geq \eta(\eta+\gamma+\omega)$ in panel (b). 